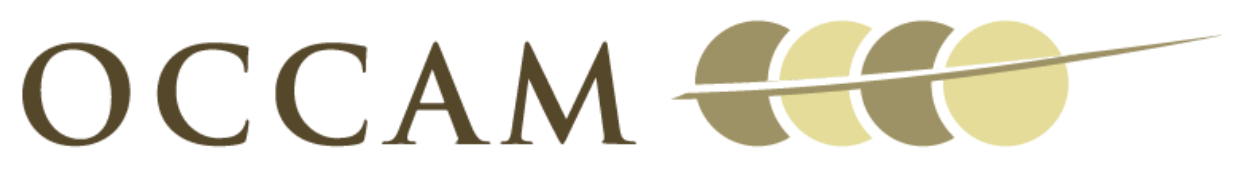

OXFORD CENTRE FOR COLLABORATIVE APPLIED MATHEMATICS

Report Number 09/19

On the existence and the applications of modified equations for stochastic differential equations

by

K.C.Zygalakis

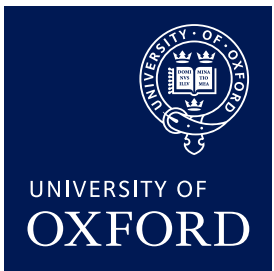

Oxford Centre for Collaborative Applied Mathematics Mathematical Institute 24 - 29 St Giles'

Oxford

OX1 3LB

England 



\title{
ON THE EXISTENCE AND THE APPLICATIONS OF MODIFIED EQUATIONS FOR STOCHASTIC DIFFERENTIAL EQUATIONS
}

\author{
K.C.ZYGALAKIS*
}

\begin{abstract}
In this paper we describe a general framework for deriving modified equations for stochastic differential equations (SDEs) with respect to weak convergence. Modified equations are derived for a variety of numerical methods, such as the Euler or the Milstein method. Existence of higher order modified equations is also discussed. In the case of linear SDEs, using the Gaussianity of the underlying solutions, we derive a SDE which the numerical method solves exactly in the weak sense. Applications of modified equations in the numerical study of Langevin equations is also discussed.
\end{abstract}

Key words. Stochastic Differential Equations, Langevin equation, weak convergence, harmonic oscillator, multiplicative noise.

AMS subject classifications. $60-08,65 \mathrm{c} 30$.

1. Introduction. This paper considers the existence of modified equations for stochastic differential equations (SDEs) with respect to weak convergence. This is essentially a form of backward error analysis; a technique which has been extremely successful in understanding the behaviour of numerical methods for ordinary differential equations (ODEs). It is possible to fit an ODE (the so called modified equation) to a numerical method to very high order accuracy. Backward error analysis has been of particular importance in the numerical study of Hamiltonian problems, since it approximates symplectic numerical methods by a perturbed Hamiltonian system, giving an approximate statistical mechanics description for symplectic methods. The reader is encouraged to see the monographs $[6,21]$ for a review and further references.

A natural question is whether such techniques extend to SDEs and in which sense. This is a very important question since, unlike the ODEs case, there exist two different notions of convergence for SDEs. In this paper we focus on deriving modified equations for SDEs with respect to weak convergence. The only prior work in this area, that the author is aware of, is [24], [22], where in [24] linear Langevin equations were considered and in [22] modified equations were derived only for the forward and backward Euler approximations to Itô SDEs with additive noise. Another interesting paper is [3], where higher order corrections for the one step probability distribution were calculated for the Euler method. The modified equations for the Euler method were used in [18] to exhibit the fact that the Euler method performs poorly for small random perturbations of Hamiltonian flows.

In this paper, using weak stochastic Taylor expansions [20], we describe a general framework for deriving modified equations for SDEs with additive and multiplicative noise, with respect to weak convergence. Actually the nature of the noise is a very subtle issue since, as we later show in the case of multiplicative noise it is impossible to write down a modified SDE that approximates the Euler method to second order accuracy. However, this problem is solved, once we consider the Milstein's method [11], where we show that it is possible to fit a SDE up to second order accuracy.

The rest of the paper is organised as follows. In Section 2, we briefly recap some basic facts regarding weak convergence for numerical approximation of SDEs and describe what is meant by a modified equation, following very closely the presentation

${ }^{*}$ Oxford Centre for Collaborative Applied Mathematics (OCCAM) (zygalakis@maths .ox .ac.uk). 
in [22]. For expository reasons the discussion is restricted to one dimensional SDEs, but the generalisation to multiple dimensions is straightforward. In Section 3, using the approach presented in Section 2, we present a general framework for deriving an arbitrary order modified equation for any first order numerical method. In Section 4 we discuss the existence of modified equations for various numerical schemes, while in Section 5 we study linear SDEs where we are able to derive a modified SDE, which the numerical method solves exactly in the weak sense. In Section 6, we discuss applications of modified equations in the numerical study of the Langevin equation, where we try to generalise some well known concepts for ODEs to the appropriate stochastic context. Finally in Section 7 we perform various numerical investigations to validate our theoretical findings.

2. Weak convergence and modified equations. Consider the following SDE

$$
d X=v(X) d t+\sigma(X) d W(t), X(0)=Y,
$$

where $v: \mathbb{R}^{d} \mapsto \mathbb{R}, \sigma: \mathbb{R}^{d \times n} \mapsto \mathbb{R}^{d}$ are smooth functions and $W(t)$ is a standard $n$ dimensional Brownian motion.

Now let $C_{P}^{l}\left(\mathbb{R}^{d}, \mathbb{R}\right)$ denote the space of $l$ times continuously differentiable functions $g: \mathbb{R}^{d} \mapsto \mathbb{R}$ which, together with their partial derivatives of orders up to and inculding order $l$, have polynomial growth. Then we have the following definition

Definition 2.1. A numerical approximation $X_{0}, X_{1}, \cdots$ of (2.1) obtained using a time step $\Delta t$, converges to $X(t)$, in the weak sense: for $T>0$,

$$
\left|\mathbb{E}\left(\phi\left(X_{n}\right)\right)-\mathbb{E}(\phi(X(n \Delta t)))\right|=\mathcal{O}\left(\Delta t^{p}\right), 0 \leq n \Delta t \leq T,
$$

for $\phi \in C_{P}^{2(p+1)}\left(\mathbb{R}^{d}, \mathbb{R}\right)$, where $p$ is known as the weak order of the method.

In the case of the test functions $\phi$ it is enough to think about polynomials of order up to $2 p+1$.

The simplest example of a weak first order method is the forward Euler method (often called the Euler-Maruyama method), given by

$$
X_{n+1}=X_{n}+v\left(X_{n}\right) \Delta t+\sqrt{\Delta t} \sigma\left(X_{n}\right) \xi_{n},
$$

where $\xi_{n}$ are i.i.d random variables with $\xi_{0} \sim \mathcal{N}(0,1)$. The reader is pointed to [11, 13] for a technical statement of the proof and a review of other approximation methods. An excellent introduction to algorithmic aspects of numerical methods for SDEs can be found in [7].

The goal is to modify the $\operatorname{SDE}(2.1)$ to define a process $\tilde{X}$ that better describes the numerical approximation $X_{n}$, in the sense that

$$
\left|\mathbb{E}\left(\phi\left(X_{n}\right)\right)-\mathbb{E}(\phi(\tilde{X}(n \Delta t)))\right|=\mathcal{O}\left(\Delta t^{p+q}\right), 0 \leq n \Delta t \leq T,
$$

where $q>0$ is the increase in the order of accuracy. We define $\tilde{X}$ as the solution to the modified Itô SDE

$$
d \tilde{X}=\left[v(\tilde{X})+\tilde{v}(\tilde{X}) \Delta t^{p}\right] d t+\left[\sigma(\tilde{X})+\tilde{\sigma}(\tilde{X}) \Delta t^{p}\right] d W(t), \tilde{X}(0)=Y,
$$

where $\tilde{v}, \tilde{\sigma}$ are smooth functions to be determined, and look for convergence at one order higher

$$
\left|\mathbb{E}\left(\phi\left(X_{n}\right)\right)-\mathbb{E}(\phi(\tilde{X}(n \Delta t)))\right|=\mathcal{O}\left(\Delta t^{p+1}\right), 0 \leq n \Delta t \leq T,
$$


The main technical fact we use in studying this problem is the following one: The $p$-th weak convergence of a numerical method can be reduced to studying the approximations over one time step $[11,13]$. This is very similar to the idea of local error for ordinary differential equations [10], where the global error in the numerical approximation can essentially be determined using only the local error. More precisely, to achieve $p$-th order weak convergence, one would expect that we should have

$$
\left|\mathbb{E}\left(\phi\left(X_{1}\right)\right)-\mathbb{E}(\phi(X(\Delta t)))\right|=\mathcal{O}\left(\Delta t^{p+1}\right),
$$

for all test functions $\phi(x)$. Thus in order to achieve (2.6), we need

$$
\left|\mathbb{E}\left(\phi\left(X_{1}\right)\right)-\mathbb{E}(\phi(\tilde{X}(\Delta t)))\right|=\mathcal{O}\left(\Delta t^{p+2}\right),
$$

for all test functions $\phi$. It turns out (Theorem 14.5.2 in [11], Theorem 9.1 in [13]) that in order to achieve weak order $p$-convergence it is sufficient for (2.7) to be satisfied for all the polynomials $\phi(x)$ up to degree $2 p+1$, and thus for $(2.8)$ to be satisfied for all the polynomials $\phi(x)$ of degree up to $2 p+3$.

REMARK 2.1. In [22], this fact was used in order to derive modified equation for the Euler-Maruyama method. Our approach is slightly more different, since we study conditions in order for (2.7), (2.8) to be satisfied for all $\phi$, and not just for the sufficient polynomials. Even though this seems more complicated it "provides us" with a general framework in which we are able derive modified equations for a wider class of numerical methods.

3. A general framework. In this section we describe a general framework for deriving modified equations of arbitrary order with respect to weak convergence for SDEs. As we have previously discussed in order to fit a modified SDE to a numerical method of weak order $p$ we need (2.8) to be satisfied, which implies that the important thing is to understand what happens to expectations of functionals of the path $X(t)$ given by (2.1). We do this using weak Taylor expansions [20].

3.1. Weak Taylor Expansion. There exist a natural way of looking at expectations of functionals of the path for SDEs and that is using the backward Kolmogorov equation [19]. More precisely, we can associate an Itô SDE with a partial differential operator $\mathcal{L}_{0}$, which is called the generator of the SDE in the relevant literature [17]. In the case of $(2.1)(n=d=1), \mathcal{L}_{0}$ is given by

$$
\mathcal{L}_{0} u:=v(x) \frac{d u}{d x}+\frac{1}{2} \sigma^{2}(x) \frac{d^{2} u}{d x^{2}} .
$$

The backward Kolmogorov equation associated with the SDE (2.1) is

$$
\begin{aligned}
\frac{\partial u}{\partial t} & =\mathcal{L}_{0} u, \\
u(x, 0) & =\phi(x),
\end{aligned}
$$

where $\mathcal{L}_{0}$ given by (3.1). The probabilistic way [17, 15] of looking at solutions of this equation is that

$$
u(x, t)=\mathbb{E}(\phi(x(t)) \mid x(0)=x) .
$$

We now integrate equation (3.2) to obtain

$$
u(x, \Delta t)-u(x, 0)=\mathcal{L}_{0} \int_{0}^{\Delta t} u(x, s) d s .
$$


Assuming $u(x, t)$ is $N+1$ times differentiable with respect to t we have the following Taylor expansion

$$
u(x, s)=u(x, 0)+s \frac{\partial u(x, 0)}{\partial s}+\cdots+\frac{s^{N}}{N !} \frac{\partial^{N} u(x, 0)}{\partial s^{N}}+R .
$$

Using this together with equation (3.3) we obtain

$$
u(x, \Delta t)-u(x, 0)=\Delta t \mathcal{L}_{0} u(x, 0)+\sum_{k=1}^{N} \Delta t^{k+1} \mathcal{L}_{0} \frac{\partial^{k} u(x, 0)}{\partial s^{k}}+\mathcal{O}\left(\Delta t^{N+2}\right)
$$

Now using the fact that $u(x, 0)=\phi(x)$ together with equation (3.2) it is easy to see that

$$
\frac{\partial^{k} u(x, 0)}{\partial s^{k}}=\mathcal{L}_{0}^{k} \phi(x)
$$

We thus obtain

$$
u(x, \Delta t)-\phi(x)=\sum_{k=0}^{N} \frac{\Delta t^{k+1}}{(k+1) !} \mathcal{L}_{0}^{k+1} \phi(x)+\mathcal{O}\left(\Delta t^{N+2}\right) .
$$

REMARK 3.1. A weak p-th order method should now obey this expansion up to terms of order $\mathcal{O}\left(\Delta t^{p}\right)$, since if we then subtract the method from the expansion the local error would be $\mathcal{O}\left(\Delta t^{p+1}\right)$, which implies that the global weak error is of $p$-th order.

3.2. 1-st modified equation. Following Remark 3.1 we expect a first order numerical method to satisfy

$$
u_{\text {num }}(x, \Delta t)-\phi(x)=\Delta t \mathcal{L}_{0} \phi(x)+\Delta t^{2} \mathcal{A}_{1} \phi+\mathcal{O}\left(\Delta t^{3}\right),
$$

where $\mathcal{A}_{1}$ is a partial differential operator acting on $\phi$ that depends on the choice of the numerical method used. This implies that

$$
u(x, \Delta t)-u_{\text {num }}(x, \Delta t)=\mathcal{O}\left(\Delta t^{2}\right),
$$

so the local error is $\mathcal{O}\left(\Delta t^{2}\right)$, which implies that our numerical scheme is of weak order one.

The first order modified equation for $(2.1)$ is of the form

$$
d \tilde{X}=\left[v(\tilde{X})+v_{1}(\tilde{X}) \Delta t\right] d t+\left(\sigma(\tilde{X})+\sigma_{1}(\tilde{X}) \Delta t\right) d W(t) .
$$

The generator of this process is given by

$$
\mathcal{L}^{\Delta t} u:=\left(v(x)+\Delta t v_{1}(x)\right) \frac{d u}{d x}+\frac{1}{2}\left(\sigma(x)+\Delta t \sigma_{1}(x)\right)^{2} \frac{d^{2} u}{d x^{2}},
$$

and the associated backward Kolmogorov equation reads

$$
\begin{aligned}
\frac{\partial u}{\partial t} & =\mathcal{L}^{\Delta t} u, \\
u(x, 0) & =\phi(x) .
\end{aligned}
$$


We now write the generator of this modified equation in the more convenient form

$$
\mathcal{L}^{\Delta t}:=\mathcal{L}_{0}+\Delta t \mathcal{L}_{1}+\Delta t^{2} \mathcal{L}_{2}
$$

where

$$
\begin{aligned}
& \mathcal{L}_{0} u:=v(x) \frac{d u}{d x}+\frac{\sigma^{2}(x)}{2} \frac{d^{2} u}{d x^{2}}, \\
& \mathcal{L}_{1} u:=v_{1}(x) \frac{d u}{d x}+\sigma(x) \sigma_{1}(x) \frac{d^{2} u}{d x^{2}}, \\
& \mathcal{L}_{2} u:=\frac{\sigma_{1}^{2}(x)}{2} \frac{d^{2} u}{d x^{2}} .
\end{aligned}
$$

Note that $\mathcal{L}_{0}$ is the generator of the original SDE (2.1). Using the same procedure as before together with (3.7) we obtain:

$$
\begin{aligned}
u_{\text {mod }}(x, \Delta t)-\phi(x) & =\Delta t \mathcal{L}_{0} \phi(x)+\Delta t^{2} \mathcal{L}_{1} \phi(x)+\Delta t^{3} \mathcal{L}_{2} \phi(x) \\
& +\frac{\Delta t^{2}}{2} \mathcal{L}_{0} \mathcal{L}^{\Delta t} \phi(x)+\frac{\Delta t^{3}}{2} \mathcal{L}_{1} \mathcal{L}^{\Delta t} \phi(x)+\frac{\Delta t^{4}}{2} \mathcal{L}_{2} \mathcal{L}^{\Delta t} \phi(x)
\end{aligned}
$$

We can now subtract $u_{\text {num }}$ from $u_{\text {mod }}$ to obtain:

$$
u_{\text {mod }}(x, \Delta t)-u_{\text {num }}(x, \Delta t)=\Delta t^{2}\left(\mathcal{L}_{1} \phi(x)+\frac{1}{2} \mathcal{L}_{0}^{2} \phi(x)-\mathcal{A}_{1} \phi(x)\right)+\mathcal{O}\left(\Delta t^{3}\right)
$$

So in order for equation (3.5) to be $\mathcal{O}\left(\Delta t^{2}\right)$ apart from the numerical approximation we need

$$
\mathcal{L}_{1} \phi(x)=\mathcal{A}_{1} \phi(x)-\frac{1}{2} \mathcal{L}_{0}^{2} \phi(x), \forall \phi
$$

REMARK 3.2. Equation (3.9) is true in arbitrary dimensions and not just for one dimension. The only difference is that the associated partial differential operators would be slightly more complicated, which as we later see makes the calculations slightly more involved.

REMARK 3.3. We could have used the representation $u(x, t)=e^{\mathcal{L} t} \phi(x)$ to represent the solution of the backward-Kolmogorov equation and then expand it using the formula for the exponential of an operator. We would have obtained the same expansion as in (3.4). We choose however to use a Taylor expansion instead in order to make a connection with ODEs. More precisely, in the case of ODEs the first order modified equation would be obtained by (3.9), for $\phi(x)=x$. This fact illustrates the complexity of SDEs, since in order to obtain modified equations we need (3.9) to be satisfied for every $\phi$ in the space of smooth functions and not just for $\phi(x)=x$, as it is the case with ODEs

REMARK 3.4. A similar local error analysis related with the chemical master equation can be found in [9]. Finally in [23], a similar in nature expansion for the global weak error was given in terms of the time step $\Delta t$ for the Euler and the Milstein method. 
3.3. Higher Order Modified Equations. In this section we describe what are the conditions that should be satisfied in order to derive higher order modified equations. We start by describing analytically the case of a second order modified equation for a first order method. More precisely, a second order modified SDE for a first order method would be of the form

$$
d \tilde{X}=\left[v(\tilde{X})+\Delta t v_{1}(\tilde{X})+\Delta t^{2} v_{2}(\tilde{X})\right]+\left[\sigma(\tilde{X})+\Delta t \sigma_{1}(\tilde{X})+\Delta t^{2} \sigma_{2}(\tilde{X})\right] d W(t),
$$

where $v_{1}(x), \sigma_{1}(x)$ are the modified coefficients we had determine before and $v_{2}(x), \sigma_{2}(x)$ are the new coefficients to be determined. The generator $\mathcal{L}^{\Delta t}$ of this SDE is of the form:

$$
\mathcal{L}^{\Delta t}:=\mathcal{L}_{0}+\Delta t \mathcal{L}_{1}+\Delta t^{2} \mathcal{L}_{2}+\Delta t^{3} \mathcal{L}_{3}+\Delta t^{4} \mathcal{L}_{4}
$$

where

$$
\begin{aligned}
\mathcal{L}_{0} u & :=v(x) \frac{d u}{d x}+\frac{\sigma^{2}(x)}{2} \frac{d^{2} u}{d x^{2}}, \\
\mathcal{L}_{1} u & :=v_{1}(x) \frac{d u}{d x}+\sigma(x) \sigma_{1}(x) \frac{d^{2} u}{d x^{2}}, \\
\mathcal{L}_{2} u & :=v_{2}(x) \frac{d u}{d x}+\left(\frac{\sigma_{1}^{2}(x)}{2}+\sigma(x) \sigma_{2}(x)\right) \frac{d^{2} u}{d x^{2}}, \\
\mathcal{L}_{3} u & :=\sigma_{1}(x) \sigma_{2}(x) \frac{d^{2} u}{d x^{2}} \\
\mathcal{L}_{4} u & :=\frac{\sigma_{2}^{2}(x)}{2} \frac{d^{2} u}{d x^{2}} .
\end{aligned}
$$

Using the same procedure as in Section 3.1 together with (3.11) we obtain:

$$
\begin{aligned}
u_{\text {mod }}(x, \Delta t)-\phi(x) & =\Delta t \mathcal{L}_{0} \phi \\
& +\Delta t^{2}\left(\mathcal{L}_{1} \phi+\frac{1}{2} \mathcal{L}_{0}^{2} \phi\right) \\
& +\Delta t^{3}\left(\mathcal{L}_{2} \phi+\frac{1}{2} \mathcal{L}_{0} \mathcal{L}_{1} \phi+\frac{1}{2} \mathcal{L}_{1} \mathcal{L}_{0} \phi+\frac{1}{6} \mathcal{L}_{0}^{3} \phi\right)+\mathcal{O}\left(\Delta t^{4}\right) .
\end{aligned}
$$

Since our numerical method is of first order we have:

$$
u_{\text {num }}(x, \Delta t)-\phi(x)=\Delta t \mathcal{L}_{0} \phi(x)+\Delta t^{2} \mathcal{A}_{1} \phi+\Delta t^{3} \mathcal{A}_{2} \phi+\mathcal{O}\left(\Delta t^{4}\right) .
$$

By following the same technique as in Section 3.1 and assuming that (3.9) holds, the condition for (3.10) to be $\mathcal{O}\left(\Delta t^{3}\right)$ away from our first order numerical method is

$$
\mathcal{L}_{2} \phi=\mathcal{A}_{2} \phi-\frac{1}{2}\left(\mathcal{L}_{1} \mathcal{L}_{0} \phi+\mathcal{L}_{0} \mathcal{L}_{1} \phi\right)-\frac{1}{6} \mathcal{L}_{0}^{3} \phi, \forall \phi
$$

It is easy to see that by repeating this procedure we can derive conditions for modified equations of arbitrary high order. More precisely the $n$-th modified equation for a first order method would be of the form

$$
d \tilde{X}=\left[v(\tilde{X})+\sum_{k=1}^{n} \Delta t^{k} v_{k}(\tilde{X})\right] d t+\left[\sigma(\tilde{X})+\sum_{k=1}^{n} \Delta t^{k} \sigma_{k}(\tilde{X})\right] d W(t)
$$


where $v_{k}(x), \sigma_{k}(x), k=1, \cdots n-1$ are the modified coefficients we had determine before and $v_{n}(x), \sigma_{n}(x)$ are the new coefficients to be determined. The generator $\mathcal{L}^{\Delta t}$ of (3.18) is of the form

$$
\mathcal{L}^{\Delta t}:=\mathcal{L}_{0}+\sum_{k}^{2 n} \Delta t^{k} \mathcal{L}_{k}
$$

and $\mathcal{L}_{n}$ contains the unknown coefficients $v_{n}(x), \sigma_{n}(x)$. Using the same procedure as in Section 3.1 together with (3.19) we obtain:

$$
\begin{aligned}
u_{\text {mod }}(x, \Delta t)-\phi(x) & =\Delta t \mathcal{L}_{0} \phi \\
& +\Delta t^{2}\left(\mathcal{L}_{1} \phi+\frac{1}{2} \mathcal{L}_{0}^{2} \phi\right) \\
& +\Delta t^{3}\left(\mathcal{L}_{2} \phi+\frac{1}{2} \mathcal{L}_{0} \mathcal{L}_{1} \phi+\frac{1}{2} \mathcal{L}_{1} \mathcal{L}_{0} \phi+\frac{1}{6} \mathcal{L}_{0}^{3} \phi\right) \\
& \vdots \\
& +\Delta t^{n+1}\left(\mathcal{L}_{n} \phi+\frac{1}{2}\left(\mathcal{L}_{0} \mathcal{L}_{n-1} \phi+\mathcal{L}_{n-1} \mathcal{L}_{0} \phi\right)+\cdots+\frac{1}{n !} \mathcal{L}_{0}^{n} \phi\right) \\
& +\mathcal{O}\left(\Delta t^{n+2}\right) .
\end{aligned}
$$

Since our numerical method is of first order we have:

$$
u_{n u m}(x, \Delta t)-\phi(x)=\Delta t \mathcal{L}_{0} \phi(x)+\sum_{k=1}^{n} \Delta t^{k+1} \mathcal{A}_{k} \phi+\mathcal{O}\left(\Delta t^{n+2}\right)
$$

By following the same technique as in Section 3.1 and assuming that all the conditions up to the (n-1)-th modified equation hold, the condition for (3.18) to be $\mathcal{O}\left(\Delta t^{n+1}\right)$ away from our first order numerical method is

$$
\mathcal{L}_{n} \phi=\mathcal{A}_{n} \phi-\frac{1}{2}\left(\mathcal{L}_{0} \mathcal{L}_{n-1} \phi+\mathcal{L}_{n-1} \mathcal{L}_{0} \phi\right)-\cdots-\frac{1}{n !} \mathcal{L}_{0}^{n} \phi, \forall \phi
$$

4. Different numerical methods and existence of associated modified equations. In this section we discuss the existence of modified equations for different weak first order methods. We use the word existence, since as we later show there would be cases where even to derive a modified SDE that fits the numerical method to second order would be impossible. We split our investigations into two parts, namely in one and in multiple dimensions.

4.1. One dimension. In this subsection we discuss the existence of modified equations for one dimensional SDEs. As we have seen in Section 3, in order to calculate the modified equations we need to know the structure of $\mathcal{L}_{0}$ and $\mathcal{A}_{1}$, with the later being method specific. However, $\mathcal{L}_{0}$ is associated with the $\operatorname{SDE}(2.1)$ and it is not method dependent. After some calculations, which can be found in Appendix 
A, we find that:

$$
\begin{aligned}
\mathcal{L}_{0}^{2} f & =\left(v(x) v^{(1)}(x)+\frac{\sigma^{2}(x)}{2} v^{(2)}(x)\right) \phi^{(1)}(x) \\
& +\left(v^{2}(x)+\sigma^{2}(x) v^{(1)}(x)+v(x) \sigma(x) \sigma^{(1)}(x)+\frac{\sigma^{2}(x)}{2}\left(\sigma^{(1)}(x)\right)^{2}+\frac{\sigma^{3}(x)}{2} \sigma^{(2)}(x)\right) \phi^{(2)}(x) \\
& +\left(\sigma^{3}(x) \sigma^{(1)}(x) f^{(3)}(x)+\sigma^{2}(x) v(x)\right) \phi^{(3)}(x) \\
& +\frac{\sigma^{4}(x)}{4} \phi^{(4)}(x),
\end{aligned}
$$

where by $\phi^{(i)}, v^{(i)}, \sigma^{(i)}$, we denote the $i$-th derivative of the associated function. We are now ready to study issues regarding the existence of modified equations for different numerical methods.

4.1.1. Euler-Maruyama method. The one step approximation for the EulerMaruyama method [11] is given by

$$
x(\Delta t)=x+v(x) \Delta t+\sigma(x) \sqrt{\Delta t} \xi,
$$

where $\xi$ is $\mathcal{N}(0,1)$ distributed. It is easy to see that

$$
\mathbb{E}(\phi(x(\Delta t)) \mid x(0)=x)=\mathbb{E}(\phi(x+v(x) \Delta t+\sigma(x) \sqrt{\Delta t} \xi)) .
$$

After taking the Taylor expansion of $\phi$ up to 4-th order we find that

$$
\begin{aligned}
\mathbb{E}(\phi(x(\Delta t)) \mid x(0)=x) & =\phi(x)+\Delta t \mathcal{L}_{0} \phi \\
& +\Delta t^{2}\left(\frac{v^{2}(x) \phi^{(2)}(x)}{2}+\frac{3 \sigma^{2}(x) v(x)}{6} \phi^{(3)}(x)+\frac{3 \sigma^{4}(x)}{24} \phi^{(4)}(x)\right) \\
& +\mathcal{O}\left(\Delta t^{3}\right) .
\end{aligned}
$$

The details of this calculation can be found in Appendix A. From equation (4.2) we can deduce that

$$
\mathcal{A}_{1} \phi=\frac{v^{2}(x) \phi^{(2)}(x)}{2}+\frac{3 \sigma^{2}(x) v(x)}{6} \phi^{(3)}(x)+\frac{3 \sigma^{4}(x)}{24} \phi^{(4)}(x) .
$$

Using this, together with (4.1), equation (3.9) becomes

$$
\begin{aligned}
\mathcal{L}_{1} \phi(x) & =-\frac{1}{2}\left(v(x) v^{(1)}(x)+\frac{\sigma^{2}(x)}{2} v^{(2)}(x)\right) \phi^{(1)}(x) \\
& +-\frac{1}{2}\left(\sigma^{2}(x) v^{(1)}(x)+v(x) \sigma(x) \sigma^{(1)}(x)+\frac{\sigma^{2}(x)}{2}\left(\sigma^{(1)}(x)\right)^{2}+\frac{\sigma^{3}(x)}{2} \sigma^{(2)}(x)\right) \phi^{(2)}(x) \\
& +\frac{\sigma^{3}(x)}{2} \sigma^{(1)}(x) \phi^{(3)}(x) .
\end{aligned}
$$

There is a problem, however, with equation (4.3) since $\mathcal{L}_{1}$ is a second order differential operator and the right hand side of (4.3) contains third derivatives in $\phi$. This implies that in the case of Euler method for SDEs with multiplicative noise it is impossible to write down a modified Itô SDE that fits the numerical method to second weak order. We later see that if instead of the Euler method we use the Milstein's method 
such a derivation of a modified Itô SDE is possible, under appropriate smoothness assumptions for the drift $v(x)$ and the diffusion coefficient $\sigma(x)$.

Another way of getting around this problem is to consider $(2.1)$ for $\sigma(x)=\sigma$. In that case (4.3) reads

$$
\mathcal{L}_{1} \phi(x)=-\left(\frac{1}{2} v(x) v^{(1)}(x)+\frac{\sigma^{2}}{4} v^{(2)}(x)\right) \phi^{(1)}(x)-\frac{\sigma^{2}}{2} v^{(1)} \phi^{(2)}(x) .
$$

Using the expression for $\mathcal{L}_{1}$ we can deduce

$$
\begin{aligned}
v_{1}(x) & =-\left(\frac{1}{2} v(x) v^{(1)}(x)+\frac{\sigma^{2}}{4} v^{(2)}(x)\right), \\
\sigma_{1}(x) & =-\frac{\sigma}{2} v^{(1)}(x),
\end{aligned}
$$

which agrees with the modified equations derived in [22] for the Euler-Maruyama method. However, besides the case where $v(x)$ is linear, the modified equation (2.5) is an Itô SDE with multiplicative noise and we thus cannot iterate it to obtain the next higher order modified equation.

4.1.2. Milstein's method. The Milstein's method for the approximation of $x(\Delta t)[11]$ reads

$$
x(\Delta t)=x+v(x) \Delta t+\sigma(x) \sqrt{\Delta t} \xi+\frac{1}{2} \sigma(x) \sigma^{(1)}(x)\left(\Delta t \xi^{2}-\Delta t\right),
$$

where $\xi$ is $\mathcal{N}(0,1)$ distributed. It is easy to see that

$$
\mathbb{E}(\phi(x(\Delta t)) \mid x(0)=x)=\mathbb{E}\left(\phi\left(x+v(x) \Delta t+\sigma(x) \sqrt{\Delta t} \xi+\frac{1}{2} \sigma(x) \sigma^{(1)}(x)\left(\Delta t \xi^{2}-\Delta t\right)\right)\right) .
$$

After taking the Taylor expansion of $\phi$ up to 4-th order we find that

$$
\begin{aligned}
\mathbb{E}(\phi(x(\Delta t)) \mid x(0)=x) & =\phi(x)+\Delta t \mathcal{L}_{0} \phi \\
& +\frac{\Delta t^{2}}{2}\left(v^{2}(x)+\frac{1}{2} \sigma(x) \sigma^{(1)}(x)\right) \phi^{(2)}(x) \\
& +\frac{3 \Delta t^{2}}{6}\left(\sigma^{3}(x) \sigma^{(1)}(x)+\sigma^{(2)}(x) v(x)\right) \phi^{(3)}(x) \\
& +\frac{3 \Delta t^{2}}{24} \sigma^{4}(x) \phi^{(4)}(x)+\mathcal{O}\left(\Delta t^{3}\right) .
\end{aligned}
$$

The details of this calculation can be found in the Appendix A. From equation (4.2) we can deduce that

$$
\mathcal{A}_{1} \phi=\frac{v^{2}(x) \phi^{(2)}(x)}{2}+\frac{1}{2}\left(\sigma^{3}(x) \sigma^{(1)}(x)+\sigma^{(2)}(x) v(x)\right) \phi^{(3)}(x)+\frac{3 \sigma^{4}(x)}{24} \phi^{(4)}(x) .
$$

Using this, together with (4.1), equation (3.9) becomes

$$
\begin{aligned}
\mathcal{L}_{1} \phi(x) & =-\frac{1}{2}\left(v(x) v^{(1)}(x)+\frac{\sigma^{2}(x)}{2} v^{(2)}(x)\right) \phi^{(1)}(x) \\
& -\frac{1}{2}\left(\sigma^{2}(x) v^{(1)}(x)+v(x) \sigma(x) \sigma^{(1)}(x)+\frac{\sigma^{2}(x)}{2}\left(\sigma^{(1)}(x)\right)^{2}+\frac{\sigma^{3}(x)}{2} \sigma^{(2)}(x)\right) \phi^{(2)}(x) \\
& +\frac{1}{4} \sigma(x) \sigma^{(1)}(x) \phi^{(2)}(x) .
\end{aligned}
$$


We thus have

$$
\begin{aligned}
v_{1}(x) & =-\frac{1}{2}\left(v(x) v^{(1)}(x)+\frac{\sigma^{2}(x)}{2} v^{(2)}(x)\right) \\
\sigma(x) \sigma_{1}(x) & =-\frac{1}{2}\left(\sigma^{2}(x) v^{(1)}(x)+v(x) \sigma(x) \sigma^{(1)}(x)+\frac{\sigma^{2}(x)}{2}\left(\sigma^{(1)}(x)\right)^{2}+\frac{\sigma^{3}(x)}{2} \sigma^{(2)}(x)\right) \\
& +\frac{1}{4} \sigma(x) \sigma^{(1)}(x),
\end{aligned}
$$

and thus for the Milstein's method the modified coefficients are

$$
\begin{aligned}
& v_{1}(x)=-\frac{1}{2}\left(v(x) v^{(1)}(x)+\frac{\sigma^{2}(x)}{2} v^{(2)}(x)\right) \\
& \sigma_{1}(x)=-\frac{1}{2}\left(\sigma(x) v^{(1)}(x)+v(x) \sigma^{(1)}(x)+\frac{\sigma(x)}{2}\left(\sigma^{(1)}(x)\right)^{2}+\frac{\sigma^{2}(x)}{2} \sigma^{(2)}(x)-\frac{1}{2} \sigma^{(1)}(x)\right) .
\end{aligned}
$$

A good reality check for these coefficients is checking that they agree with the EulerMaruyama method in the case where the noise is additive, since then the EulerMaruyama coincides with the Milstein's method. This is indeed the case, since if we set $\sigma(x)=\sigma$ equation (4.6) becomes equation (4.4). Another important thing to notice is that in principle it is possible to iterate (2.5) to find a higher order modified equation.

4.2. Multiple dimensions. In this subsection we discuss the existence of modified equations for multiple dimensional SDEs. For calculation reasons we restrict our attention to the following SDE

$$
d X=v(X) d t+\Sigma d W(t)
$$

where $X \in \mathbb{R}^{d}, \Sigma \in \mathbb{R}^{d \times d}$ and $W(t)$ is a standard $d$-dimensional Brownian motion. As before $\mathcal{L}_{0}^{2}$ is not method specific, so after some calculations, which are presented in Appendix B, we find that, in the case where $\Sigma$ is a constant matrix:

$$
\begin{aligned}
\mathcal{L}_{0}^{2} \phi & =v_{k} \partial_{k} v_{i} \partial_{i} \phi+v_{k} v_{i} \partial_{k} \partial_{i} \phi+\frac{1}{2} v_{k}\left(\Sigma \Sigma^{T}\right)_{i j} \partial_{k} \partial_{i} \partial_{j} \phi \\
& +\frac{1}{2}\left(\Sigma \Sigma^{T}\right)_{k m}\left[\partial_{k} \partial_{m} v_{i} \partial_{i} \phi+\partial_{m} v_{i} \partial_{k} \partial_{i} \phi+\partial_{k} v_{i} \partial_{m} \partial_{i} \phi+v_{i} \partial_{k} \partial_{m} \partial_{i} \phi\right] \\
& +\frac{1}{4}\left(\Sigma \Sigma^{T}\right)_{k m}\left(\Sigma \Sigma^{T}\right)_{i j} \partial_{k} \partial_{m} \partial_{i} \partial_{j} \phi
\end{aligned}
$$

where we have used Einstein's summation formula and $k, m, i, j=1, \cdots, d$, while $\phi: \mathbb{R}^{d} \mapsto \mathbb{R}$ is a smooth test function. The first order modified equation for (4.7) is of the form

$$
d \tilde{X}=\left[v(\tilde{X})+\Delta t v_{1}(\tilde{X})\right] d t+\left[\Sigma+\Delta t \Sigma_{1}(\tilde{X})\right] d W(t)
$$

and thus in this case

$$
\mathcal{L}_{1}=\tilde{v} \cdot \nabla+\frac{1}{2}\left(\Sigma_{1} \Sigma^{T}+\Sigma \Sigma_{1}^{T}\right): \nabla \nabla
$$


4.2.1. Euler-Maruyama method. The one step approximation for the Euler Maruyama method for (4.7) is given by

$$
x(\Delta t)=x+\Delta t v(x)+\Sigma \sqrt{\Delta t} \xi,
$$

with the understanding that $\xi$ is a $\mathcal{N}\left(0, I_{d}\right)$ distributed random variable. By taking the Taylor expansion of $\phi$ up to 4 -th order as we have done before, we find

$$
\begin{aligned}
\mathbb{E}(\phi(x(\Delta t)) \mid x(0)=x) & =\phi(x)+\Delta t \mathcal{L}_{0} \phi \\
& +\frac{\Delta t^{2}}{2} v_{i} v_{j} \partial_{i} \partial_{j} \phi \\
& +\frac{3 \Delta t^{2}}{6}\left(\Sigma \Sigma^{T}\right)_{k m} v_{i} \partial_{k} \partial_{m} \partial_{i} \phi \\
& +\frac{3 \Delta t^{2}}{24}\left(\Sigma \Sigma^{T}\right)_{k m}\left(\Sigma \Sigma^{T}\right)_{i j} \partial_{k} \partial_{m} \partial_{i} \partial_{j} \phi+\mathcal{O}\left(\Delta t^{3}\right) .
\end{aligned}
$$

The details of this calculation can be found in Appendix B. From equation (4.12) we deduce that

$$
\begin{aligned}
\mathcal{A}_{1} \phi & =\frac{\Delta t^{2}}{2} v_{i} v_{j} \partial_{i} \partial_{j} \phi \\
& +\frac{3 \Delta t^{2}}{6}\left(\Sigma \Sigma^{T}\right)_{k m} v_{i} \partial_{k} \partial_{m} \partial_{i} \phi \\
& +\frac{3 \Delta t^{2}}{24}\left(\Sigma \Sigma^{T}\right)_{k m}\left(\Sigma \Sigma^{T}\right)_{i j} \partial_{k} \partial_{m} \partial_{i} \partial_{j} \phi
\end{aligned}
$$

Using this, together with (4.8), equation (3.9) becomes

$$
\begin{aligned}
\mathcal{L}_{1} \phi & =\left(-\frac{1}{2} v_{k} \partial_{k} v_{i}-\frac{1}{4}\left(\Sigma \Sigma^{T}\right)_{k m} \partial_{k} \partial_{m} v_{i}\right) \partial_{i} \phi \\
& -\frac{1}{4}\left(\Sigma \Sigma^{T}\right)_{k m}\left(\partial_{m} v_{i} \partial_{k} \partial_{i} \phi+\partial_{k} v_{i} \partial_{m} \partial_{i} \phi\right) .
\end{aligned}
$$

Thus the modified drift $\tilde{v}$ is given by

$$
v_{1}=-\frac{1}{2}(\nabla v) v-\frac{1}{4}\left(\Sigma \Sigma^{T}\right): \nabla \nabla \mathbf{v},
$$

and the modified diffusion coefficient satisfies the Lyapunov equation

$$
\Sigma_{1} \Sigma^{T}+\Sigma \Sigma_{1}^{T}=-\Sigma \Sigma^{T} \nabla v .
$$

In the case where $\Sigma=\sigma I$ the resulting $v_{1}, \Sigma_{1}$ are the same as the ones found in [22].

4.2.2. Symplectic Euler. We now study the following symplectic Euler method

$$
\begin{aligned}
& q_{n+1}=q_{n}+\Delta t p_{n+1}, \\
& p_{n+1}=p_{n}-\left(\gamma p_{n}+V^{\prime}\left(q_{n}\right)\right) \Delta t+\sigma \sqrt{\Delta t} \xi_{n},
\end{aligned}
$$

which corresponds to solving the following Langevin equation

$$
\begin{aligned}
& d q=p d t, \\
& d p=-\left(\gamma p+V^{\prime}(q)\right) d t+\sigma d W(t),
\end{aligned}
$$


where $W(t)$ is a one standard dimensional Brownian motion. This equation describes the motion of a particle in the potential $V(q)$, subject to linear friction and molecular diffusion $[4,19]$, the magnitude of which is given by $\gamma$ and $\sigma$ respectively. The reason that we consider solving (4.14) with the symplectic Euler method described by (4.13) is that in the absence of noise and friction $(\sigma=\gamma=0)$, (4.14) describes a Hamiltonian system and thus a symplectic method is preferred. There exist a number of interesting publications on the interplay between symplectic methods for ODE and their extension to stochastic problems $[14,16]$.

Now assuming that we have started from $p, q$, using (4.13), the numerical approximation for $q(\Delta t), p(\Delta t)$ is given by

$$
\begin{aligned}
& q(\Delta t)=q+p \Delta t-\gamma p \Delta t^{2}-V^{\prime}(q) \Delta t^{2}+\sigma \sqrt{\Delta t^{3}} \xi \\
& p(\Delta t)=p-\left(\gamma p+V^{\prime}(q)\right) \Delta t+\sigma \sqrt{\Delta t} \xi .
\end{aligned}
$$

By taking the Taylor expansion of $\phi$ up to 4-th order as we have done before we find:

$$
\begin{aligned}
\mathbb{E}(\phi(p(\Delta t), q(\Delta t)) \mid & (p(0), q(0)))=(p, q))=\phi(p, q)+\Delta t \mathcal{L}_{0} \phi \\
- & \Delta t^{2}\left(\gamma p+V^{\prime}(q)\right) \partial_{q} \phi \\
+ & \frac{\Delta t^{2}}{2}\left(p^{2} \partial_{q}^{2} \phi+2\left(\sigma^{2}-p\left(\gamma p+V^{\prime}(q)\right)\right) \partial_{p} \partial_{q} \phi+\left(\gamma p+V^{\prime}(q)\right)^{2} \partial_{p}^{2} \phi\right) \\
+ & \frac{\Delta t^{2}}{6}\left(3 \sigma^{2} p \partial_{q} \partial_{p}^{2} \phi-3 \sigma^{2}\left(\gamma p+V^{\prime}(q)\right) \partial_{p}^{3} \phi\right)+\frac{3 \Delta t^{2} \sigma^{4}}{24} \partial_{p}^{4} \phi \\
+ & \mathcal{O}\left(\Delta t^{3}\right) .
\end{aligned}
$$

The details of this calculation can be found in Appendix B. From equation (4.15) we can deduce that:

$$
\begin{aligned}
\mathcal{A}_{1} \phi & =-\left(\gamma p+V^{\prime}(q)\right) \partial_{q} \phi+\frac{1}{2}\left(p^{2} \partial_{q}^{2} \phi+2\left(\sigma^{2}-p\left(\gamma p+V^{\prime}(q)\right)\right) \partial_{p} \partial_{q} \phi+\left(\gamma p+V^{\prime}(q)\right)^{2} \partial_{p}^{2} \phi\right) \\
& +\frac{1}{6}\left(3 \sigma^{2} p \partial_{q} \partial_{p}^{2} \phi-3 \sigma^{2}\left(\gamma p+V^{\prime}(q)\right) \partial_{p}^{3} \phi\right)+\frac{3 \sigma^{4}}{24} \partial_{p}^{4} \phi .
\end{aligned}
$$

Using this, together with (4.8), equation (3.9) becomes:

$$
\mathcal{L}_{1} \phi=-\frac{1}{2}\left(V^{\prime}(q)+\gamma p\right) \partial_{q} \phi-\frac{1}{2}\left(\gamma V^{\prime}(q)+\gamma^{2} p-p V^{\prime \prime}(q)\right) \partial_{p} \phi+\frac{\sigma^{2}}{2} \partial_{p} \partial_{q} \phi+\frac{1}{2} \gamma \sigma^{2} \partial_{p}^{2} \phi
$$

which implies that the modified equation is of the form:

$$
\begin{aligned}
& d q=\left[p-\frac{\Delta t}{2}\left(V^{\prime}(q)+\gamma p\right)\right] d t+\sigma \frac{\Delta t}{2} d W(t) \\
& d p=\left[-\gamma p-V^{\prime}(q)-\frac{\Delta t}{2}\left(\gamma V^{\prime}(q)+\gamma^{2} p-p V^{\prime \prime}(q)\right)\right] d t+\sigma\left(1+\frac{\Delta t}{2} \gamma\right) d W(t) .
\end{aligned}
$$

It is important to note that the noise that drives the $q$-equation is the same as the noise that drives the $p$-equation. Again a good reality check for this modified equation is to set $\sigma=\gamma=0$ and then we obtain

$$
\begin{aligned}
& d p=\tilde{H}_{p}(p, q) d t, \\
& d q=-\tilde{H}_{q}(p, q) d t,
\end{aligned}
$$


where

$$
\tilde{H}(p, q)=\frac{1}{2} p^{2}+V(q)-\frac{\Delta t}{2} V^{\prime}(q) p
$$

is the modified Hamiltonian that we would have obtained using modified equations for the corresponding ODE [6].

4.2.3. 1st order Lie-Trotter splitting method. We now study a 1st order Lie-Trotter splitting method. This method is used in order to solve Langevin equations of the form

$$
\begin{aligned}
d q & =H_{p} d t, \\
d p & =-\left(H_{q}+\gamma p\right) d t+\sigma d W(t) .
\end{aligned}
$$

The main idea behind this method is that we split (4.18) into two parts. The first one is the one we would obtain in the absence of noise, namely

$$
\begin{aligned}
& d q=H_{p} d t, \\
& d p=-H_{q} d t,
\end{aligned}
$$

while the second one is an Ornstein-Uhlenbeck for the generalised momentum $p$,

$$
\begin{aligned}
d q & =0, \\
d p & =-\gamma p d t+\sigma d W(t) .
\end{aligned}
$$

Then we can solve (4.19) using a first order symplectic method, while we can solve (4.20) exactly. The numerical approximation is then given by the composition of solutions of these two equations. The main property of this method [16] is that preserves the Boltzmann-Gibbs distribution in the appropriate norm up to the order of the symplectic method used to solve (4.19).

For this study we choose the first order symplectic method to be the symplectic Euler method. The numerical approximation in this case is given by:

$$
\begin{aligned}
& q_{n+1}=q^{*}+\Delta t p_{n+1}, \\
& p_{n+1}=p^{*}-V^{\prime}\left(q^{*}\right) \Delta t,
\end{aligned}
$$

where $\left(q^{*}, p^{*}\right)$ satisfy

$$
\begin{aligned}
& q^{*}=q_{n}, \\
& p^{*}=e^{-\gamma \Delta t} p_{n}+\beta \xi_{n},
\end{aligned}
$$

where $\xi_{n}$ is $\mathcal{N}(0,1)$ distributed and

$$
\beta^{2}=\frac{\sigma^{2}}{2 \gamma}\left(1-e^{-2 \gamma \Delta t}\right)
$$

Thus the one step approximation starting from $(q, p)$ is given by:

$$
\begin{aligned}
& q(\Delta t)=q+\Delta t p(\Delta t), \\
& p(\Delta t)=e^{-\gamma \Delta t} p-V^{\prime}(q) \Delta t+\beta \xi,
\end{aligned}
$$


where $\xi$ is $\mathcal{N}(0,1)$ distributed. By taking the Taylor expansion of $\phi$ up to 4 -th order as we have done before we find:

$$
\begin{aligned}
\mathbb{E}(\phi(p(\Delta t), q(\Delta t)) \mid & (p(0), q(0)))=(p, q))=\phi(p, q)+\Delta t \mathcal{L}_{0} \phi \\
- & \Delta t^{2}\left[\left(\gamma p+V^{\prime}(q)\right) \partial_{q} \phi-\frac{\gamma^{2}}{2} p \partial_{p} \phi\right] \\
+ & \frac{\Delta t^{2}}{2}\left(p^{2} \partial_{q}^{2} \phi+2\left(\sigma^{2}-p\left(\gamma p+V^{\prime}(q)\right)\right) \partial_{p} \partial_{q} \phi+\left(\gamma p+V^{\prime}(q)\right)^{2} \partial_{p}^{2} \phi\right) \\
+ & \frac{\Delta t^{2}}{6}\left(3 \sigma^{2} p \partial_{q} \partial_{p}^{2} \phi-3 \sigma^{2}\left(\gamma p+V^{\prime}(q)\right) \partial_{p}^{3} \phi\right)+\frac{3 \Delta t^{2} \sigma^{4}}{24} \partial_{p}^{4} \phi \\
+ & \mathcal{O}\left(\Delta t^{3}\right) .
\end{aligned}
$$

The details of this calculation can be found in Appendix B. From equation (4.22) we can deduce that:

$$
\begin{aligned}
\mathcal{A}_{1} \phi & =-\left(\gamma p+V^{\prime}(q)\right) \partial_{q}+\frac{\gamma^{2}}{2} p \partial_{p} \\
& +\frac{1}{2}\left(p^{2} \partial_{q}^{2} \phi+2\left(\sigma^{2}-p\left(\gamma p+V^{\prime}(q)\right)\right) \partial_{p} \partial_{q} \phi+\left(\gamma p+V^{\prime}(q)\right)^{2} \partial_{p}^{2} \phi\right) \\
& +\frac{1}{6}\left(3 \sigma^{2} p \partial_{q} \partial_{p}^{2} \phi-3 \sigma^{2}\left(\gamma p+V^{\prime}(q)\right) \partial_{p}^{3} \phi\right)+\frac{3 \sigma^{4}}{24} \partial_{p}^{4} \phi .
\end{aligned}
$$

Using this, together with (4.8), equation (3.9) becomes:

$$
\begin{aligned}
\mathcal{L}_{1} \phi & =-\frac{1}{2}\left(V^{\prime}(q)+\gamma p\right) \partial_{q} \phi-\frac{1}{2}\left(\gamma V^{\prime}(q)-p V^{\prime \prime}(q)\right) \partial_{p} \phi \\
& +\frac{\sigma^{2}}{2} \partial_{p} \partial_{q} \phi+\frac{1}{2} \gamma \sigma^{2} \partial_{p}^{2} \phi
\end{aligned}
$$

which implies that the modified equation is of the form:

$$
\begin{aligned}
& d q=\left[p-\frac{\Delta t}{2}\left(V^{\prime}(q)+\gamma p\right)\right] d t+\sigma \frac{\Delta t}{2} d W(t) \\
& d p=\left[-\gamma p-V^{\prime}(q)-\frac{\Delta t}{2}\left(\gamma V^{\prime}(q)-p V^{\prime \prime}(q)\right)\right] d t+\sigma\left(1+\frac{\Delta t}{2} \gamma\right) d W(t)
\end{aligned}
$$

It is important to note that the noise that drives the q-equation is the same as the noise that drives the $p$-equation. Again a good reality check for this modified equation is to set $\sigma=\gamma=0$ and then we obtain:

$$
\begin{aligned}
& d p=\tilde{H}_{p}(p, q) d t, \\
& d q=-\tilde{H}_{q}(p, q) d t,
\end{aligned}
$$

where

$$
\tilde{H}(p, q)=\frac{1}{2} p^{2}+V(q)-\frac{\Delta t}{2} V^{\prime}(q) p
$$

is the modified Hamiltonian that we would have obtained using modified equations for the corresponding ODE [6].

REMARK 4.1. It is important to note that both of the modified equations (4.16), (4.23) have no longer the structure of the Langevin equation (4.14). The implications of this fact are futherly discussed in Section 6 . 
5. Linear SDEs and $\infty$-modified equation. In this section we study the case of linear SDEs. More precisely, we show that we can write down a perturbation of the original SDE, which the numerical solution of the original SDE solves exactly in the weak sense. This is a well known fact in the case of Runge-Kutta methods for linear ODEs [1] and it was used in [21] in order to derive a modified Hamiltonian system for the case of the harmonic oscillator, which the numerical solution for the symplectic Euler method solves exactly. From now on we shall use the term $\infty$ modified SDE for the modified SDE, which the numerical method solves exactly in the weak sense.

We now apply these ideas in the case of linear SDEs. More precisely let $x \in \mathbb{R}^{d}$ satisfying the following linear SDE:

$$
d x=A x d t+\Sigma d W_{t}
$$

where $A, \Sigma$ are $d \times d$ matrices. Then it is possible to show using the variation of constants formula that (5.1) has the solution [4]:

$$
x(t)=e^{A t} x(0)+\int_{0}^{t} e^{A(t-s)} \Sigma d W_{s} .
$$

Since this is a linear system the numerical approximation $\tilde{x}(\Delta t)$ of $x(\Delta t)$ by any numerical method, assuming that we start from $x(0)=x$ would be of the form:

$$
x(\Delta t)=A(\Delta t) x+f(\Delta t, \omega),
$$

where $f(\Delta t, \omega)$ denotes the way we approximate the noise and $A(\Delta t)$ is another matrix for which $A(0)=I$. For example in the case of Euler-Maruyama method

$$
\begin{aligned}
A(\Delta t) & =(I+\Delta t A), \\
f(\Delta t, \omega) & =\Sigma \sqrt{\Delta t} \xi,
\end{aligned}
$$

where $\xi$ is $\mathcal{N}\left(0, I_{d}\right)$ distributed.

The important thing to note in the case of linear SDEs is that the solution of them due to linearity is always Gaussian. This is very useful, since in order to find the $\infty$ modified SDE it is enough to check that its first and second moments over one step approximation agree exactly with those of the numerical method. Thus let the $\infty$ modified equation be of the form

$$
d x=\tilde{A} x d t+\tilde{\Sigma} d W_{t} .
$$

Then from (5.2) we have that:

$$
\begin{aligned}
\mathbb{E}(x(\Delta t) \mid x(0) & =x)=e^{\tilde{A} \Delta t} x, \\
\mathbb{E}\left(x(\Delta t) x^{T}(\Delta t) \mid x(0)\right. & =x)=e^{\tilde{A} t} x x^{T} e^{\tilde{A}^{T} t}+\int_{0}^{\Delta t} e^{\tilde{A}(t-s)} \tilde{\Sigma} \tilde{\Sigma}^{T} e^{\tilde{A}^{T}(t-s)} d s .
\end{aligned}
$$

On the other hand using (5.3) it is easy to see that:

$$
\begin{aligned}
\mathbb{E}(x(\Delta t) \mid x(0) & =x)=A(\Delta t) x, \\
\mathbb{E}\left(x(\Delta t) x^{T}(\Delta t) \mid x(0)\right. & =x)=A(\Delta t) x x^{T} A(\Delta t)^{T}+\mathbb{E}\left(f f^{T}\right) .
\end{aligned}
$$

Thus the coefficients $\tilde{\Sigma}, \tilde{A}$ of the $\infty$-modified equation satisfy

$$
\begin{aligned}
e^{\tilde{A} \Delta t} x & =A(\Delta t) x \\
e^{\tilde{A} t} x x^{T} e^{\tilde{A}^{T} t}+\int_{0}^{\Delta t} e^{\tilde{A}(t-s)} \tilde{\Sigma} \tilde{\Sigma}^{T} e^{\tilde{A}^{T}(t-s)} d s & =A(\Delta t) x x^{T} A(\Delta t)^{T}+\mathbb{E}\left(f f^{T}\right) .
\end{aligned}
$$


We can solve for $\tilde{A}$ to obtain:

$$
\tilde{A}=\frac{\log (A(\Delta t))}{\Delta t}
$$

while $\tilde{\Sigma}$ satisfies:

$$
\int_{0}^{\Delta t} e^{\tilde{A}(t-s)} \tilde{\Sigma} \tilde{\Sigma}^{T} e^{\tilde{A}^{T}(t-s)} d s=\mathbb{E}\left(f f^{T}\right) .
$$

After some calculations that are presented in Appendix D equation (5.6) becomes

$$
e^{\tilde{A} \Delta t} \tilde{\Sigma} \tilde{\Sigma}^{T} e^{\tilde{A}^{T} \Delta t}-\tilde{\Sigma} \tilde{\Sigma}^{T}=\tilde{A} J+J \tilde{A}^{T}
$$

where $J=\mathbb{E}\left(f f^{T}\right)$. We also need to note that, since (5.3) solves (5.1) numerically then

$$
\lim _{\Delta t \rightarrow 0} \tilde{A}=A .
$$

5.1. Connection with ODEs. In this subsection we make a connection between the $\infty$ modified equations for the SDEs described before and the ODEs governing the evolution of the mean and the covariance. More precisely, let $M(t)=\mathbb{E}(x(t))$ and $\sigma(t)=\operatorname{Cov}(x(t), x(t))$. Then by using Itô's formula and taking expectation we find that $M(t), \sigma(t)$ satisfy the following ODEs

$$
\begin{aligned}
\frac{d M}{d t} & =-A M, \\
\frac{d \sigma}{d t} & =-A \sigma-\sigma A^{T}+\Sigma \Sigma^{T} .
\end{aligned}
$$

Similarly the mean and the covariance of the modified equation $\tilde{M}(t), \tilde{\sigma}(t)$ satisfy the following ODEs

$$
\begin{aligned}
& \frac{d \tilde{M}}{d t}=-\tilde{A} \tilde{M} \\
& \frac{d \tilde{\sigma}}{d t}=-\tilde{A} \tilde{\sigma}-\tilde{\sigma} \tilde{A}^{T}+\tilde{\Sigma} \tilde{\Sigma}^{T}
\end{aligned}
$$

It is important to see that equations for the modified mean and covariance (5.9a), (5.9b) are the $\infty$ modified equations that we would have obtained for the ODEs (5.8a),(5.8b), using the numerical approximation (5.3) for $(5.1)[1,6]$.

6. Backward Error Analysis for the Langevin Equation. In this section we discuss how we can extent the backward error analysis for deterministic Hamiltonian systems to the case of the Langevin equation. In the case of deterministic time autonomous Hamiltonian systems

$$
\begin{aligned}
d q & =H_{p} d t, \\
d p & =-H_{q} d t,
\end{aligned}
$$

it is easy to see that since $\partial_{t} H(p, q)=0$

$$
\frac{d H}{d t}=0,
$$


thus the solutions of (6.1) evolve so as for the Hamiltonian $H(p, q)$ to remain constant. It is thus naturally expected that an appropriate numerical discretization for (6.1) would respect in some sense the properties of (6.1). It is then possible to show using backward error analysis [6, 21] that there exist numerical schemes which conserve up to high accuracy a modified Hamiltonian $H_{\Delta t}(p, q)$, which is close to the original Hamiltonian $H(p, q)$.

6.1. Properties of the Langevin equation. A natural question is how does equation (6.2) carry forward in the stochastic setting of the Langevin equation (4.14). It is very important to note that the Langevin equation (4.14) can be written in the following form

$$
\begin{aligned}
& d q=H_{p} d t \\
& d p=-\left(H_{q}+\gamma H_{p}\right) d t+\sigma d W(t),
\end{aligned}
$$

for $H(p, q)=\frac{1}{2} p^{2}+V(q)$. The reason for writing equation (4.14) in the form of $(6.3)$ is that the invariant measure of (6.3) is given by:

$$
\pi_{0}(p, q)=e^{-\frac{2 \gamma}{\sigma^{2}} H(p, q)},
$$

where we have to assume appropriate growth for the Hamiltonian at infinity, in order for the invariant measure to be normalizable.

We now use Itô's formula for (4.14) to see that

$$
H(p(t), q(t))=H(p(0), q(0))+\int_{0}^{t} \mathcal{L}_{0} H(q(s), p(s)) d s+\frac{\sigma^{2}}{2} \int_{0}^{t} \partial_{p} H(q(s), p(s)) d W_{s},
$$

where $\mathcal{L}_{0}$ is the generator of the process given by

$$
\mathcal{L}_{0}:=p \partial_{q}-\left(\gamma p+V^{\prime}(q)\right) \partial_{p}+\frac{\sigma^{2}}{2} \partial_{p}^{2}
$$

If we take expectations the stochastic integral vanishes and thus

$$
\mathbb{E}(H(p(t), q(t)))=\mathbb{E}(H(p(0), q(0)))+\mathbb{E}\left(\int_{0}^{t} \mathcal{L}_{0} H(q(s), p(s)) d s\right) .
$$

We now have

$$
\mathbb{E}\left(\int_{0}^{t} \mathcal{L}_{0} H(q(s), p(s)) d s\right)=\int_{0}^{t}\left(\int_{\mathbb{R}^{2}} \mathcal{L}_{0} H(p, q) \pi(p, q, s) d p d q\right) d s
$$

where $\pi$ satisfies the Fokker-Planck equation with initial data $\pi(p, q, 0)=\rho_{0}$. Using that $\mathcal{L}_{0}^{*}$ is the $L^{2}$ adjoint of $\mathcal{L}_{0}$ we obtain

$$
\int_{\mathbb{R}^{2}} \mathcal{L}_{0} H(p, q) \pi(p, q, s) d p d q=\int_{\mathbb{R}^{2}} H(p, q) \mathcal{L}_{0}^{*} \pi(p, q, s) d p d q=0,
$$

Now, since our system is a Hamiltonian one, it is natural to assume that the initial conditions are distributed according to the Boltzmann-Gibbs distribution $\pi_{0}$ given by (6.4), satisfying

$$
\mathcal{L}_{0}^{*} \pi_{0}=0
$$


which implies that $\pi(p, q, s)=\pi_{0}(p, q), \forall s>0$. Thus using (6.8) and (6.7) we obtain

$$
\mathbb{E}(H(p(t), q(t)))=\mathbb{E}(H(p(0), q(0))),
$$

which implies that when starting from stationarity the mean Hamiltonian remains constant. This is the analogous of the conservation of the Hamiltonian in the case of no friction and noise. In this sense a suitable numerical method for the Langevin equation (4.14), just as in the case of ODEs, would be one which preserves up to high accuracy the average of a modified Hamiltonian $H_{\Delta t}(p, q)$ which is close to the original Hamiltonian $H(p, q)$.

From the point of view of backward error analysis if such a method exists, this would imply that the first modified equation would be of the form (6.3) for some modified Hamiltonian $H_{1}(p, q)$ which differs from the original Hamiltonian $\mathcal{O}(\Delta t)$. It is important to note that neither the symplectic Euler method nor the 1st order Lie-Trotter splitting method belong to this class of methods, since their modified equations (4.16),(4.23) are not in the form of (6.3), since for both of them the $q$ variable is driven by noise. In the next subsection we construct a numerical scheme which 1st modified equation is in the form of (6.3).

6.2. 1st order integrated Euler. In this subsection we describe the way we derive a numerical scheme for (4.14), which its 1st modified equation is in the form of (6.3). It is possible to solve up to quadrature (4.14) to obtain $[26,18]$ :

$$
\begin{aligned}
& q(t)=q(0)+\frac{1}{\gamma}\left(1-e^{-\gamma t}\right) p(0)-\frac{1}{\gamma} \int_{0}^{t}\left(1-e^{-\gamma(t-s)}\right) V^{\prime}(q(s)) d s+\frac{\sigma}{\gamma} \int_{0}^{t}\left(1-e^{-\gamma(t-s)}\right) d W_{s}, \\
& p(t)=p(0) e^{-\gamma t}-\int_{0}^{t} e^{-\gamma(t-s)} V^{\prime}(q(s)) d s+\sigma \int_{0}^{t} e^{-\gamma(t-s)} d W_{s} .
\end{aligned}
$$

The most straightforward numerical approximation of (6.10) is

$$
\begin{aligned}
& q_{n+1}=q_{n}+\frac{1}{\gamma}\left(1-e^{-\gamma \Delta t}\right) p_{n}-\Delta t \frac{1}{\gamma}\left(1-e^{-\gamma \Delta t}\right) V^{\prime}\left(q_{n}\right)+\alpha \xi_{n}+\delta \psi_{n}, \\
& p_{n+1}=e^{-\gamma \Delta t} p_{n}-\Delta t e^{-\gamma \Delta t} V^{\prime}\left(q_{n}\right)+\beta \xi_{n},
\end{aligned}
$$

where $\xi_{n}, \psi_{n}$ are $\mathcal{N}(0,1)$ distributed, independent random variables and the coefficients $\alpha, \delta, \beta$ are given by [18]:

$$
\begin{aligned}
\alpha^{2}+\delta^{2} & =\frac{\sigma^{2}}{\gamma^{2}}\left[\Delta t-\frac{2}{\gamma}\left(1-e^{-\gamma \Delta t}\right)+\frac{1}{2 \gamma}\left(1-e^{-2 \gamma \Delta t}\right)\right], \\
\alpha \beta & =\frac{\sigma^{2}}{2 \gamma^{2}}\left(1-e^{-\gamma \Delta t}\right)^{2}, \\
\beta^{2} & =\frac{\sigma^{2}}{2 \gamma}\left(1-e^{-2 \gamma \Delta t}\right) .
\end{aligned}
$$

From now on, because of the way we approximated the integrals, we will call this method 1st order integrated Euler. The one step time approximation of this method assuming that $(q(0), p(0))=(q, p)$ is given by:

$$
\begin{aligned}
& q(\Delta t)=q+\frac{1}{\gamma}\left(1-e^{-\gamma \Delta t}\right) p-\Delta t \frac{1}{\gamma}\left(1-e^{-\gamma \Delta t}\right) V^{\prime}(q)+\alpha \xi+\delta \psi, \\
& p(\Delta t)=e^{-\gamma \Delta t} p-\Delta t e^{-\gamma \Delta t} V^{\prime}(q)+\beta \xi .
\end{aligned}
$$


By taking the Taylor expansion of $\phi$ up to 4-th order as we have done before we find

$$
\begin{aligned}
\mathbb{E}(\phi(p(\Delta t), q(\Delta t)) \mid & (p(0), q(0)))=(p, q))=\phi(p, q)+\Delta t \mathcal{L}_{0} \phi \\
- & \Delta t^{2}\left[\left(\frac{\gamma p}{2}+V^{\prime}(q)\right) \partial_{q} \phi-\frac{\gamma^{2}}{2} p \partial_{p} \phi\right] \\
+ & \frac{\Delta t^{2}}{2}\left(p^{2} \partial_{q}^{2} \phi+\left(\sigma^{2}-2 p\left(\gamma p+V^{\prime}(q)\right)\right) \partial_{p} \partial_{q} \phi+\left(\gamma p+V^{\prime}(q)\right)^{2} \partial_{p}^{2} \phi\right) \\
+ & \frac{\Delta t^{2}}{6}\left(3 \sigma^{2} p \partial_{q} \partial_{p}^{2} \phi-3 \sigma^{2}\left(\gamma p+V^{\prime}(q)\right) \partial_{p}^{3} \phi\right)+\frac{3 \Delta t^{2} \sigma^{4}}{24} \partial_{p}^{4} \phi .
\end{aligned}
$$

The details for this calculation can be found in Appendix C. From equation (6.14) we can deduce that

$$
\begin{aligned}
\mathcal{A}_{1} \phi & =-\left(\frac{\gamma p}{2}+V^{\prime}(q)\right) \partial_{q}+\frac{\gamma^{2}}{2} p \partial_{p} \\
& +\frac{1}{2}\left(p^{2} \partial_{q}^{2} \phi+\left(\sigma^{2}-2 p\left(\gamma p+V^{\prime}(q)\right)\right) \partial_{p} \partial_{q} \phi+\left(\gamma p+V^{\prime}(q)\right)^{2} \partial_{p}^{2} \phi\right) \\
& +\frac{1}{6}\left(3 \sigma^{2} p \partial_{q} \partial_{p}^{2} \phi-3 \sigma^{2}\left(\gamma p+V^{\prime}(q)\right) \partial_{p}^{3} \phi\right)+\frac{3 \sigma^{4}}{24} \partial_{p}^{4} \phi .
\end{aligned}
$$

Using this, together with (4.8), equation (3.9) becomes

$$
\mathcal{L}_{1} \phi=-\frac{1}{2} V^{\prime}(q) \partial_{q} \phi-\frac{1}{2}\left(\gamma V^{\prime}(q)-p V^{\prime \prime}(q)\right) \partial_{p} \phi+\frac{1}{2} \gamma \sigma^{2} \partial_{p}^{2} \phi,
$$

which implies that the modified equation is of the form

$$
\begin{aligned}
& d q=\left[p-\frac{\Delta t}{2} V^{\prime}(q)\right] d t \\
& d p=\left[-\gamma p-V^{\prime}(q)-\frac{\Delta t}{2}\left(\gamma V^{\prime}(q)-p V^{\prime \prime}(q)\right)\right] d t+\sigma\left(1+\frac{\Delta t}{2} \gamma\right) d W(t)
\end{aligned}
$$

If we now consider the modified Hamiltonian

$$
\tilde{H}_{1}(p, q)=\frac{1}{2} p^{2}+V(q)-\frac{\Delta t}{2} V^{\prime}(q) p,
$$

we can write the modified equation (4.16) in the more convenient form

$$
\begin{aligned}
& d q=\tilde{H}_{1 p} d t \\
& d p=-\left(\tilde{H}_{1 q}+\gamma \tilde{H}_{1 p}\right) d t+\sigma\left(1+\frac{\Delta t}{2} \gamma\right) d W(t),
\end{aligned}
$$

and using a similar analysis like the original Hamiltonian equation we see that

$$
\mathbb{E}\left(\tilde{H}_{1}(p(t), q(t))\right)=\mathbb{E}\left(\tilde{H}_{1}(p(0), q(0))\right),
$$

which implies that the modified Hamiltonian $\tilde{H}_{1}$ given by (6.16) does not change on average, when starting from stationarity.

REMARK 6.1. It is important to notice that in the deterministic case $(\sigma, \gamma=0)$ the 1st order integrated Euler method, the 1st order Lie Trotter splitting method and the symplectic Euler method are exactly the same, and thus they have the same modified equation. However, in the stochastic case their modified equations are different. This illustrates a very important point, namely that the way we approximate the noise plays a very important role in the structure of the modified equation. 
7. Numerical Investigations. In this section we investigate numerically our theoretical findings for different numerical methods. We start by investigating linear SDEs in one and two dimensions, such as the one dimensional Ornstein-Uhlenbeck process and the harmonic oscillator. For these SDEs we compare the numerically computed moments with the $\infty$-modified equation ones. We finally study an example of an SDE driven by multiplicative noise and compare the numerical computed moments with the ones of the 1-st modified equation.

7.1. One dimensional Ornstein-Uhlenbeck process. The one dimensional Ornstein Uhlenbeck process satisfies the SDE

$$
d x=-\gamma x d t+\sigma d W_{t} .
$$

7.1.1. Forward Euler. In the case of forward Euler method it is not difficult to see that for the OU process

$$
\begin{aligned}
A(\Delta t) & =(1-\gamma \Delta t), \\
f(\Delta t, \omega) & =\sigma \sqrt{\Delta t} \xi,
\end{aligned}
$$

where $\xi$ is a $\mathcal{N}(0,1)$ distributed random variable, and we need to impose the condition $\Delta t<1 / \gamma$ in order for the numerical approximation to be stable. We can now solve (5.5), (5.7) to obtain:

$$
\begin{aligned}
& \tilde{A}=\frac{\log (1-\gamma \Delta t)}{\Delta t}, \\
& \tilde{\Sigma}=\sigma \sqrt{\frac{2 \log (1-\gamma \Delta t)}{(1-\gamma \Delta t)^{2}-1}} .
\end{aligned}
$$

Due to the linearity of the numerical approximation, it is possible to calculate explicitly the parameters of the invariant measure for the numerical approximation. More precisely, we find that the invariant measure $\rho_{\infty, \Delta t}$ is a Gaussian one, with mean 0 and variance $\sigma_{1}$ given by [25]:

$$
\sigma_{1}=\frac{\sigma^{2}}{2 \gamma-\gamma^{2} \Delta t} .
$$

This is exactly the same value for the variance we would have obtained if we used the $\infty$ modified equation we have just calculated for the forward Euler method. In Figure 7.1 we plot the numerically computed variance for different time steps and we compare it with the variance predicted by the 1 st and the $\infty$ modified equation at large times. The values of the parameters used for Figure 7.1 are $\gamma=\sigma=1$, while for the numerical solution we have used final integration time $T=50$ and $N=10^{5}$ realizations of the noise. It is obvious that the variance of the numerical approximation agrees with the one predicted by the $\infty$ modified equation. The variance calculated with the use of the first modified equation is a good approximation for the variance only for small time steps.

7.1.2. Backward Euler. In the case of backward Euler method it is not difficult to see that for the OU process

$$
\begin{aligned}
A(\Delta t) & =\frac{1}{(1+\gamma \Delta t)}, \\
f(\Delta t, \omega) & =\sigma \sqrt{\Delta t} \xi,
\end{aligned}
$$




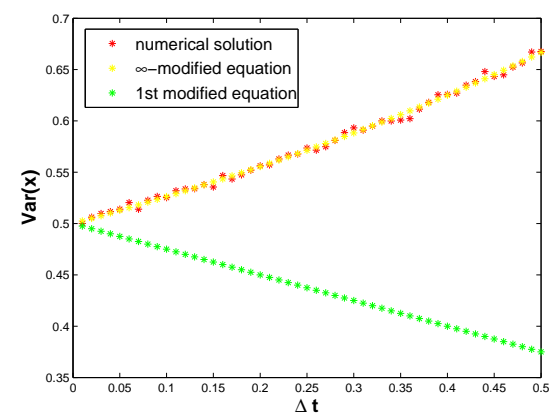

FIG. 7.1. Numerical computed variance for different time steps

where $\xi$ is a $\mathcal{N}(0,1)$ distributed random variable. It is very important to note that unlike the forward Euler method we do not need to impose any condition on the timestep $\Delta t$ in order for the numerical solution not to blow up. This is related with the fact that the backward Euler method is an implicit method. The effectiveness of implicit methods for stiff stochastic problems is discussed in [12].

We can now solve (5.5), (5.7) to obtain:

$$
\begin{aligned}
& \tilde{A}=-\frac{\log (1+\gamma \Delta t)}{\Delta t} \\
& \tilde{\Sigma}=\sigma \sqrt{\frac{2 \log (1+\gamma \Delta t)}{1-(1+\gamma \Delta t)^{-2}}} .
\end{aligned}
$$

Again due to the linearity of the numerical approximation, it is possible to calculate explicitly the parameters of the invariant measure More precisely, we find that the invariant measure $\rho_{\infty, \Delta t}$ is a Gaussian one, with mean 0 and variance $\sigma_{2}$ given by [25]:

$$
\sigma_{2}=\frac{\sigma^{2}(1+\gamma \Delta t)^{2}}{2 \gamma+\gamma^{2} \Delta t}
$$

This is exactly the same value for the variance we would have obtained if we used the $\infty$ modified equation we have just calculated for the backward Euler method. In Figure 7.2 we plot the numerical computed variance for different time steps and we compare it with the variance ${ }^{1}$ predicted by the 1 st and the $\infty$ modified equation at large times. The values of the parameters used for Figure 7.2 are $\gamma=\sigma=1$, while for the numerical solution we have used final integration time $T=50$ and $N=10^{5}$ realizations of the noise. It is obvious that the variance of the numerical approximation agrees with the one predicted by the $\infty$ modified equation. The variance calculated with the use of the first modified equation is a good approximation for the variance only for small time steps.

\footnotetext{
${ }^{1}$ In this paper we have not computed the first modified equation for the backward Euler method, but instead use the result from [22].
} 


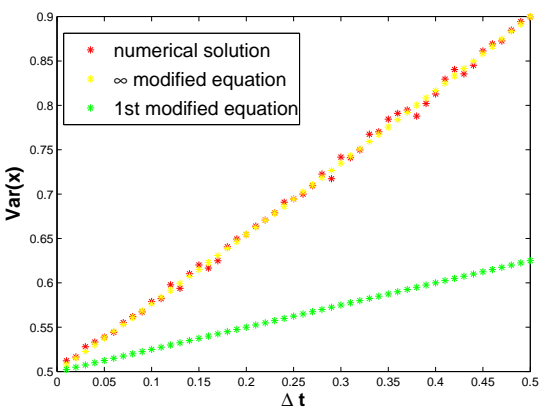

FIG. 7.2. Numerical computed variance for different time steps

7.2. Harmonic Oscillator. The harmonic oscillator in the presence of friction and noise is in the form of equation (4.14) for $H=\frac{p^{2}}{2}+\frac{q^{2}}{2}$. More precisely, we have

$$
\begin{aligned}
& d q=p d t, \\
& d p=-(q+\gamma p) d t+\sigma d W(t),
\end{aligned}
$$

The structure of the covariance matrix of the invariant measure for different numerical methods was studied in [2]. Note that we can write equation (7.6) in the form of (5.1) for

$$
A=\left(\begin{array}{cc}
0 & 1 \\
-1 & -\gamma
\end{array}\right), \Sigma=\left(\begin{array}{ll}
0 & 0 \\
0 & \sigma
\end{array}\right)
$$

7.2.1. Symplectic Euler. The symplectic Euler in the case of the harmonic oscillator reads

$$
\begin{aligned}
& q_{n+1}=q_{n}+\Delta t\left[p_{n}-\Delta t\left(\gamma p_{n}+q_{n}\right)+\sigma \sqrt{\Delta t} \xi_{n}\right] \\
& p_{n+1}=p_{n}-\Delta t\left(\gamma p_{n}+q_{n}\right)+\sigma \sqrt{\Delta t} \xi_{n},
\end{aligned}
$$

where $\xi_{n}$ is $\mathcal{N}(0,1)$ distributed. We can thus rewrite (7.8) in the form of (5.3) for

$$
A(\Delta t)=\left(\begin{array}{cc}
1-\Delta t^{2} & \Delta t-\gamma \Delta t^{2} \\
-\Delta t & 1-\gamma \Delta t
\end{array}\right)
$$

and

$$
f(\Delta t, \omega)=\left(\begin{array}{c}
\sigma \sqrt{\Delta t^{3}} \\
\sigma \sqrt{\Delta t}
\end{array}\right) \xi
$$

which implies that $\tilde{A}$ is given by:

$$
\tilde{A}=\frac{1}{\Delta t} \log \left(\begin{array}{cc}
1-\Delta t^{2} & \Delta t-\gamma \Delta t^{2} \\
-\Delta t & 1-\gamma \Delta t
\end{array}\right)
$$

We now solve the Lyapunov equation (5.7) in order to find the matrix $\tilde{\Sigma} \tilde{\Sigma}^{T}$. Using this it is possible to calculate the correlation matrix $L$ of the invariant measure using the formula [4]:

$$
L=\frac{(\operatorname{Det} A) B B^{T}+[A-(\operatorname{Tr} A) I] B B^{T}[A-(\operatorname{Tr} A) I]^{T}}{2(\operatorname{Tr} A)(\operatorname{Det} A)},
$$


where

$$
\begin{aligned}
& A=-\tilde{A}, \\
& B=\tilde{\Sigma} .
\end{aligned}
$$

In Figure 7.3 we compare the elements of the correlation matrix $L$ of the invariant measure of the $\infty$ modified equation as computed from realizations of the path and from (7.9). The values of the parameters used for Figure 7.3 are $\gamma=1, \sigma=5$, while

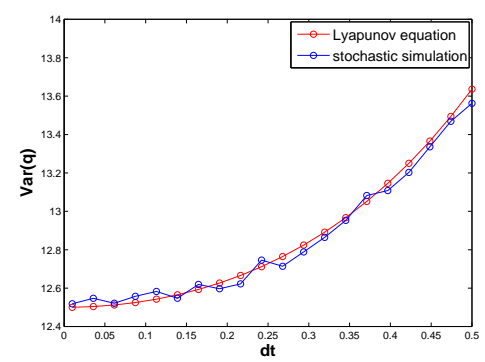

(a) $\operatorname{Var}(q)$

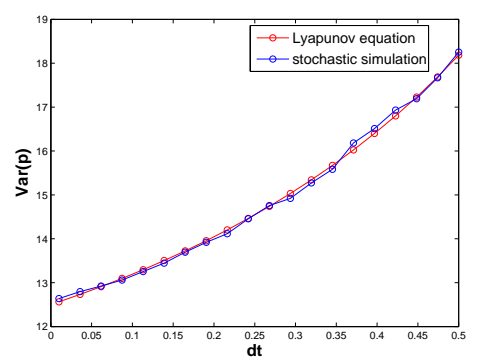

(b) $\operatorname{Var}(p)$

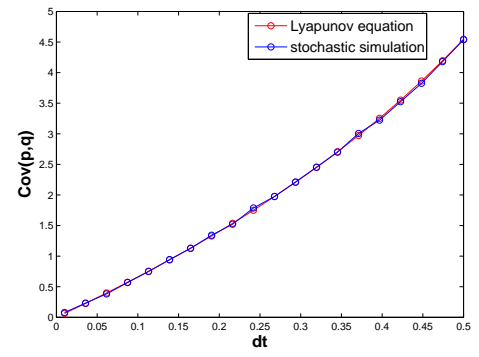

(c) $\operatorname{Cov}(p, q)$

Fig. 7.3. Correlation matrix $L$ as a function of $\Delta t$.

for the stochastic simulation we have used final integration time $T=50$ and $N=10^{5}$ realizations of the noise. As we can see there is complete agreement between the correlation matrix $L$ computed from stochastic simulations and from (7.9).

7.2.2. 1st order Lie-Trotter splitting method. The 1st order Lie-Trotter splitting method in the case of the harmonic oscillator reads

$$
\begin{aligned}
& q_{n+1}=q_{n}+\Delta t e^{-\gamma \Delta t} p_{n}-\Delta t^{2} q_{n}+\Delta t \beta \xi_{n}, \\
& p_{n+1}=e^{-\gamma \Delta t} p_{n}-\Delta t q_{n}+\beta \xi_{n},
\end{aligned}
$$

where $\xi_{n}$ is $\mathcal{N}(0,1)$ distributed. We can rewrite (7.10) in the form of (5.3) for

$$
A(\Delta t)=\left(\begin{array}{cc}
1-\Delta t^{2} & \Delta t e^{-\gamma \Delta t} \\
-\Delta t & e^{-\gamma \Delta t}
\end{array}\right)
$$

and

$$
f(\Delta t, \omega)=\left(\begin{array}{c}
\Delta t \beta \\
\beta
\end{array}\right) \xi
$$


which implies that $\tilde{A}$ is given by:

$$
\tilde{A}=\frac{1}{\Delta t} \log \left(\begin{array}{cc}
1-\Delta t^{2} & \Delta t e^{-\gamma \Delta t} \\
-\Delta t & e^{-\gamma \Delta t}
\end{array}\right) .
$$

Now as we did the case for the symplectic Euler method, we can solve the Lyapunov equation (5.7) and then use equation (7.9) to obtain the correlation matrix $L$ for the invariant measure of the $\infty$ modified equation. In Figure 7.4 we compare the elements

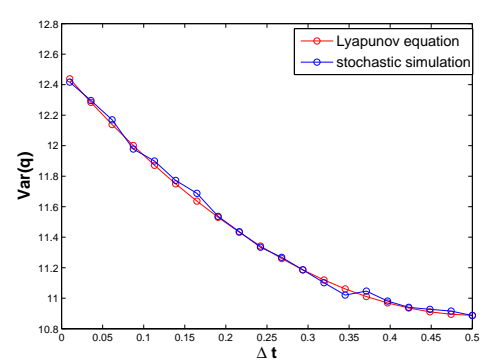

(a) $\operatorname{Var}(q)$

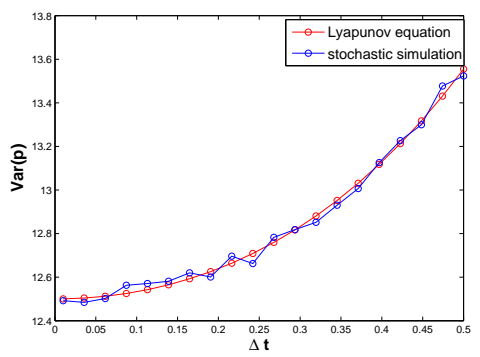

(b) $\operatorname{Var}(p)$

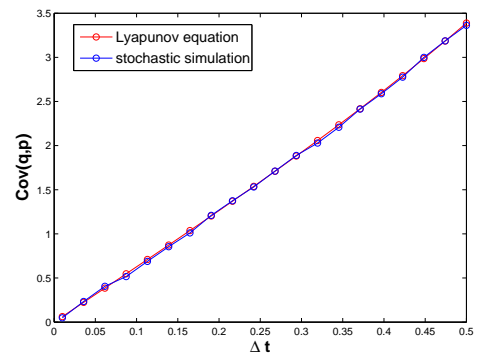

(c) $\operatorname{Cov}(p, q)$

FIG. 7.4. Correlation matrix $L$ as a function of $\Delta t$.

of the correlation matrix $L$ of the invariant measure as computed from realizations of the path and from (7.9). The values of the parameters used for Figure 7.4 are $\gamma=1, \sigma=5$, while for the stochastic simulation we have used final integration time $T=50$ and $N=10^{5}$ realizations of the noise. As we can see there is complete agreement between the correlation matrix $L$ computed from stochastic simulations and from (7.9).

Another important feature of this method is that underestimates $\operatorname{Var}(q)$, unlike the symplectic Euler, while the relative error is similar for both of the methods. On the other hand, it overestimates $\operatorname{Var}(q), \operatorname{Cov}(q, p)$ just like the symplectic Euler, but the relative size of the error in the case of the Lie-Trotter splitting method is smaller than the symplectic Euler one. This should not be a surprise, since for the Lie-Trotter splitting method, the noise in the $p$-equation is calculated exactly.

7.3. One dimensional Geometric Brownian motion. The one dimensional geometric Brownian motion $[8,15]$ satisfies the SDE

$$
d x=\mu x d t+\sigma x d W_{t} .
$$


7.3.1. Milstein Method. In (7.11) the noise is multiplicative so we use Milstein's method to solve it. Using (4.6) we have that the modified equation is

$$
d \tilde{X}=\left[\left(\mu-\frac{\Delta t}{2} \mu^{2}\right) \tilde{X}\right] d t+\left[\tilde{X}\left(\sigma-\Delta t \mu \sigma-\frac{\Delta t}{4} \sigma^{3}\right)+\frac{\Delta t}{4} \sigma\right] d W_{t} .
$$

We want to compare the first and second moments of the numerical solution with the ones of (7.11) and (7.12). For $x$ satisfying (7.11) we can calculate those moments by applying Itô's formula and then take expectations. The ODEs that we obtain are explicitly solvable and by solving them we obtain

$$
\begin{aligned}
\mathbb{E}(x(t)) & =x(0) e^{\mu t}, \\
\mathbb{E}\left(x^{2}(t)\right) & =x^{2}(0) e^{\left(2 \mu+\sigma^{2}\right) t} .
\end{aligned}
$$

In similar way we find that

$$
\mathbb{E}(\tilde{X}(t))=x(0) e^{\left(\mu-\frac{\Delta t}{2} \mu^{2}\right) t}
$$

while the second moment satisfies the following ODE:

$$
\begin{aligned}
\frac{d \mathbb{E}\left(\tilde{X}^{2}(t)\right)}{d t} & =\left[2\left(\mu-\frac{\Delta t}{2} \mu^{2}\right)+\left(\sigma-\Delta t \mu \sigma-\frac{\Delta t}{4} \sigma^{3}\right)^{2}\right] \mathbb{E}\left(\tilde{X}^{2}(t)\right) \\
& +2 \frac{\Delta t}{4} \sigma\left(\sigma-\Delta t \mu \sigma-\frac{\Delta t}{4} \sigma^{3}\right) \mathbb{E}(\tilde{X}(t))+\frac{\Delta t^{2}}{16} \sigma^{2}
\end{aligned}
$$

We can now obtain the second moment of $\tilde{X}$ by solving (7.13) using a Runge-Kutta method.

We now solve (7.11) for $\mu=2, \sigma=0.1, x(0)=1$ with the Milstein's scheme for $T=1$ using $N=510^{7}$ sample paths and five different stepsizes $\Delta t=2^{k-10}, k=$ $1, \ldots, 5$. In Figure 7.5 we plot the absolute error between the numerically computed moments and the moments of (7.11) and (7.12). As we can see the global error is of first order when we compare our numerical solution with (7.11) and second order when we compare with (7.12) as expected by theory.

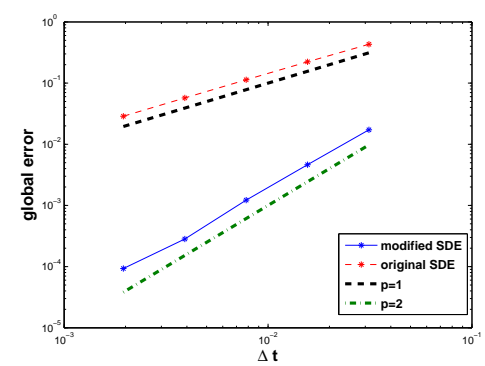

(a) First Moment

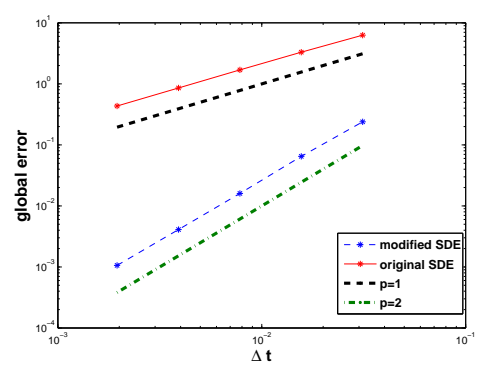

(b) Second Moment

FIG. 7.5. Global error of the numerical solution vs the original and the modified equation 
8. Conclusions and Further Work. In this paper a method for calculating modified equations for SDEs was developed building on previous ideas in [3, 20, 22, 23]. The key idea is to exploit the backward Kolmogorov equation in order to characterise the order of convergence of the numerical method and then using the same approach to find the modified SDE. A variety of numerical methods were studied in both one and multiple dimensions.

In the case of linear SDEs it was shown that it is possible to write a SDE (the $\infty$ modified equation), which the numerical method solves exactly in the weak sense. This was also verified by numerical investigations where the value of the parameters of the invariant measure of the numerical solution were compared with the ones of the $\infty$ modified equation and almost perfect agreement was found.

A backward error analysis was performed for the Langevin equation. More precisely, trying to generalise ideas of backward error analysis for Hamiltonian dynamics a numerical method was proposed, which 1-st modified equation remains a Langevin equation for a modified temperature and for the modified Hamiltonian that one obtains from the deterministic dynamics [6, 21].

There are still a lot of questions that remain open. We list some of them.

- Finding modified equations for numerical methods with respect to strong convergence.

- Understand better why there is no modified equation for the Euler-Maruyama method in the case of multiplicative noise. A possible non rigorous explanation is that the strong order of convergence of the Euler-Maruyama equation for multiplicative noise is $1 / 2$ instead of 1 as in the additive noise case, where a modified equation does exist.

- Construct higher order methods using (6.10), with similar properties to the first order integrated Euler method.

- Study the case of the generalised Langevin equation

$$
d z=(J \nabla H(z)-K \nabla H(z)) d t+\sqrt{2 \beta K} d W_{t},
$$

where $z=(p, q), J$ is a skew-symmetric matrix and $K$ is a positive definite symmetric matrix, which might possibly depend on the $q$. It can be shown that under appropriate assumptions on the Hamiltonian $H$ that the invariant measure of this system is the Boltzmann-Gibbs distribution

$$
\pi_{0}(z)=e^{-\beta^{-1} H(z)}
$$

The question is similar to what we have already studied in Section 6 is for which numerical method is the modified equation of similar form to (8.1).

- Apply a similar analysis for numerical methods used to solve the chemical Langevin equation [5].

Acknowledgements. This work was partially supported by Award No. KUKC1-013-04, made by King Abdullah University of Science and Technology (KAUST). It was also partially funded from a David Crighton Fellowship and the author would like to thank DAMTP at the University of Cambridge for kindly hosting him during his visit. The author would also like to thank J.R.Norris, T. Reis and A.M.Stuart for useful comments and suggestions and T. Lelievre for the reference [23].

Appendix A. In this appendix we present some calculations needed for the material presented in Section 4.1. We start by presenting a calculation for $\mathcal{L}_{0}^{2}$. More 
precisely we have

$$
\mathcal{L}_{0}^{2} \phi(x)=\mathcal{L}_{0}\left(v(x) \phi^{(1)}(x)+\frac{\sigma^{2}(x)}{2} \phi^{(2)}(x)\right) .
$$

We have

$$
\begin{aligned}
\mathcal{L}_{0} v(x) \phi^{(1)}(x) & =v(x) v^{(1)}(x) \phi^{(1)}(x)+v^{2}(x) \phi^{(2)}(x)+\frac{\sigma^{2}(x)}{2} v^{(2)}(x) \phi^{(1)}(x) \\
& +\sigma^{2}(x) v^{(1)}(x) \phi^{(2)}(x)+\frac{\sigma^{2}(x)}{2} v(x) \phi^{(3)}(x),
\end{aligned}
$$

and

$$
\begin{aligned}
\mathcal{L}_{0} \frac{\sigma^{2}(x)}{2} \phi^{(2)}(x) & =v(x) \frac{d}{d x}\left(\frac{\sigma^{2}(x)}{2} \phi^{(2)}(x)\right)+\frac{\sigma^{2}(x)}{2} \frac{d^{2}}{d x^{2}}\left(\frac{\sigma^{2}(x)}{2} \phi^{(2)}(x)\right) \\
& =v(x) \sigma(x) \sigma^{(1)}(x) \phi^{(2)}(x)+\frac{\sigma^{2}(x)}{2} v(x) \phi^{(3)}(x) \\
& +\frac{\sigma^{2}(x)}{2} \frac{d}{d x}\left(\sigma(x) \sigma^{(1)}(x) \phi^{(2)}(x)+\frac{\sigma^{2}(x)}{2} \phi^{(3)}(x)\right) \\
& =v(x) \sigma(x) \sigma^{(1)}(x) \phi^{(2)}(x)+\frac{\sigma^{2}(x)}{2} v(x) \phi^{(3)}(x) \\
& +\frac{\sigma^{2}(x)}{2}\left(\sigma^{(1)}(x)\right)^{2} \phi^{(2)}(x)+\frac{\sigma^{3}(x)}{2} \sigma^{(2)}(x) \phi^{(2)}(x) \\
& +\sigma^{3}(x) \sigma^{(1)}(x) \phi^{(3)}(x)+\frac{\sigma^{4}(x)}{4} \phi^{(4)}(x) .
\end{aligned}
$$

Thus $\mathcal{L}_{0}^{2}$ reads

$$
\begin{aligned}
\mathcal{L}_{0}^{2} \phi(x) & =\left(v(x) v^{(1)}(x)+\frac{\sigma^{2}(x)}{2} v^{(2)}(x)\right) \phi^{(1)}(x) \\
& +\left(v^{2}(x)+\sigma^{2}(x) v^{(1)}(x)+v(x) \sigma(x) \sigma^{(1)}(x)+\frac{\sigma^{2}(x)}{2}\left(\sigma^{(1)}(x)\right)^{2}+\frac{\sigma^{3}(x)}{2} \sigma^{(2)}(x)\right) \phi^{(2)}(x) \\
& +\left(\sigma^{3}(x) \sigma^{(1)}(x) f^{(3)}(x)+\sigma^{2}(x) v(x)\right) \phi^{(3)}(x) \\
& +\frac{\sigma^{4}(x)}{4} \phi^{(4)}(x) .
\end{aligned}
$$

A.1 Euler-Maruyama method. We present the calculations needed to find $\mathcal{A}_{1}$ for the Euler-Maruyama method in greater detail. The one step approximation for the Euler method is given by

$$
x(\Delta t)=x+v(x) \Delta t+\sigma \sqrt{\Delta t} \xi,
$$

where $\xi$ is $\mathcal{N}(0,1)$ distributed. Using the previous equation we see that

$$
\mathbb{E}(\phi(x(\Delta t)) \mid x(0)=x)=\mathbb{E}(\phi(x+v(x) \Delta t+\sigma \sqrt{\Delta t} \xi)) .
$$

We now need to take the Taylor expansion of $\phi$ up to 4 -th order so as to calculate $\mathcal{A}_{1}$. More precisely, we have

$$
\begin{aligned}
\phi(x+v(x) \Delta t+\sigma \sqrt{\Delta t} \xi) & =\phi(x)+(v(x) \Delta t+\sigma \sqrt{\Delta t} \xi) \phi^{(1)}(x)+\frac{(v(x) \Delta t+\sigma \sqrt{\Delta t} \xi)^{2}}{2} \phi^{(2)}(x) \\
& +\frac{(v(x) \Delta t+\sigma \sqrt{\Delta t} \xi)^{3}}{6} \phi^{(3)}(x)+\frac{(v(x) \Delta t+\sigma \sqrt{\Delta t} \xi)^{4}}{24} \phi^{(4)}(x)+\cdots
\end{aligned}
$$


We are interested in keeping only the $\Delta t, \Delta t^{2}$ terms. Also we need to note that the $\Delta t^{1 / 2}, \Delta t^{3 / 2}$ terms will be killed once we take expectations, since $\xi$ is a mean zero variable. We thus have:

$$
\begin{aligned}
\mathbb{E}(\phi(x(\Delta t)) \mid x(0)=x) & =\phi(x)+\Delta t \mathcal{L}_{0} \phi(x) \\
& +\Delta t^{2}\left(\frac{v^{2}(x) \phi^{(2)}(x)}{2}+\frac{3 \sigma^{2} v(x)}{6} \phi^{3}(x)+\frac{3 \sigma^{4}}{24} \phi^{(4)}(x)\right) \\
& +\mathcal{O}\left(\Delta t^{3}\right) .
\end{aligned}
$$

A.2 Milstein method. We present the calculations needed to find $\mathcal{A}_{1}$ for the Milstein's method in greater detail. The Milstein's method for the approximation of $x(\Delta t)$ in the case of multiplicative noise reads

$$
x(\Delta t)=x+v(x) \Delta t+\sigma(x) \sqrt{\Delta t} \xi+\frac{1}{2} \sigma(x) \sigma^{(1)}(x)\left(\Delta t \xi^{2}-\Delta t\right)
$$

where $\xi$ is $\mathcal{N}(0,1)$ distributed. In order to calculate $\mathcal{A}_{1}$ we need to take the Taylor expansion of $\phi$ up to 4 th order as before

$$
\begin{aligned}
\phi(x(\Delta t)) & =\phi(x)+\left(v(x) \Delta t+\sigma(x) \sqrt{\Delta t} \xi+\frac{1}{2} \sigma(x) \sigma^{(1)}(x)\left(\Delta t \xi^{2}-\Delta t\right)\right) \phi^{(1)}(x) \\
& +\frac{1}{2}\left(v(x) \Delta t+\sigma(x) \sqrt{\Delta t} \xi+\frac{1}{2} \sigma(x) \sigma^{(1)}(x)\left(\Delta t \xi^{2}-\Delta t\right)\right)^{2} \phi^{(2)}(x) \\
& +\frac{1}{6}\left(v(x) \Delta t+\sigma(x) \sqrt{\Delta t} \xi+\frac{1}{2} \sigma(x) \sigma^{(1)}(x)\left(\Delta t \xi^{2}-\Delta t\right)\right)^{3} \phi^{(3)}(x) \\
& +\frac{1}{24}\left(v(x) \Delta t+\sigma(x) \sqrt{\Delta t} \xi+\frac{1}{2} \sigma(x) \sigma^{(1)}(x)\left(\Delta t \xi^{2}-\Delta t\right)\right)^{4} \phi^{(4)}(x)+\cdots
\end{aligned}
$$

and after taking expectations and keeping terms of order up to $\Delta t^{2}$ we find

$$
\begin{aligned}
\mathbb{E}(\phi(x(\Delta t))) \mid x(0)=x) & =\Delta t\left(v(x) \phi^{(1)}(x)+\frac{\sigma^{2}(x)}{2} \phi^{(2)}(x)\right) \\
& +\frac{\Delta t^{2}}{2}\left(v^{2}(x)+\frac{1}{2} \sigma(x) \sigma^{(1)}(x)\right) \phi^{(2)}(x) \\
& +\frac{3 \Delta t^{2}}{6}\left(\sigma^{(3)}(x) \sigma^{(1)}(x)+\sigma^{(2)}(x) v(x)\right) \phi^{(3)}(x) \\
& +\frac{3 \Delta t^{2}}{24} \sigma^{4}(x) \phi^{(4)}(x) .
\end{aligned}
$$

Appendix B. In this appendix we present some calculations needed for the material presented in Section 4.2. We start by presenting a calculation for $\mathcal{L}_{0}^{2}$ in 
multiple dimensions. More precisely we have

$$
\begin{aligned}
\mathcal{L}_{0}^{2} \phi & =v(x) \cdot \nabla\left(v(x) \cdot \nabla \phi+\frac{1}{2} \Sigma \Sigma^{T}: \nabla \nabla \phi\right) \\
& +\frac{1}{2} \Sigma \Sigma^{T}: \nabla \nabla\left(v(x) \cdot \nabla \phi+\frac{1}{2} \Sigma \Sigma^{T}: \nabla \nabla \phi\right) \\
& =v_{k} \partial_{k} v_{i} \partial_{i} \phi+v_{k} v_{i} \partial_{k} \partial_{i} \phi+\frac{1}{2}\left(\Sigma \Sigma^{T}\right)_{i j} \partial_{k} \partial_{i} \partial_{j} \phi \\
& +\frac{1}{2}\left(\Sigma \Sigma^{T}\right)_{k m}\left[\partial_{k} \partial_{m} v_{i} \partial_{i} \phi+\partial_{m} v_{i} \partial_{k} \partial_{i} \phi+\partial_{k} v_{i} \partial_{m} \partial_{i} \phi+v_{i} \partial_{k} \partial_{m} \partial_{i} \phi\right] \\
& +\frac{1}{4}\left(\Sigma \Sigma^{T}\right)_{k m}\left(\Sigma \Sigma^{T}\right)_{i j} \partial_{k} \partial_{m} \partial_{i} \partial_{j} \phi .
\end{aligned}
$$

B.1 Symplectic Euler. We present the calculations needed to find $\mathcal{A}_{1}$ for the symplectic Euler in greater detail. Note, assuming we started from $p, q$, that

$$
\begin{aligned}
& q(\Delta t)=q+p \Delta t-\gamma p \Delta t^{2}-V^{\prime}(q) \Delta t^{2}+\sigma \sqrt{\Delta t^{3}} \xi, \\
& p(\Delta t)=p-\left(\gamma p+V^{\prime}(q)\right) \Delta t+\sigma \sqrt{\Delta t} \xi .
\end{aligned}
$$

So now we can take the Taylor expansion for $\phi(q(\Delta t), p(\Delta t))$ around $q, p$ up to 4 -th order. The first order term in the Taylor expansion is

$$
\left(p \Delta t-\gamma p \Delta t^{2}-V^{\prime}(q) \Delta t^{2}+\sigma \sqrt{\Delta t^{3}} \xi\right) \partial_{q} \phi+\left(-\left(\gamma p+V^{\prime}(q)\right) \Delta, t+\sqrt{\sigma \Delta t} \xi\right) \partial_{p} \phi
$$

and after taking expectation and using the fact that $\xi$ is $\mathcal{N}(0,1)$ distributed we obtain

$$
\left(p \Delta t-\gamma p \Delta t^{2}-V^{\prime}(q) \Delta t^{2}\right) \partial_{q} \phi-\left(\gamma p+V^{\prime}(q)\right) \Delta t \partial_{p} \phi
$$

The terms that are of order up to $\mathcal{O}\left(\Delta t^{2}\right)$ once we take expectations for the second order term in the Taylor expansion is

$$
\frac{1}{2}\left(\Delta t^{2} p^{2} \partial_{q}^{2} f+2\left(\sigma^{2}-p\left(\gamma p+V^{\prime}(q)\right)\right) \Delta t^{2} \partial_{p} \partial_{q} \phi+\left(\sigma^{2} \Delta t+\left(\gamma p+V^{\prime}(q)\right)^{2} \Delta t^{2}\right) \partial_{p}^{2} \phi\right) .
$$

The terms that are of order up to $\mathcal{O}\left(\Delta t^{2}\right)$ once we take expectations for the third order term in the Taylor expansion is

$$
\frac{\Delta t^{2}}{6}\left(3 \sigma^{2} p \partial_{q} \partial_{p}^{2} \phi-3 \sigma^{2}\left(\gamma p+V^{\prime}(q)\right) \partial_{p}^{3} \phi\right)
$$

and finally the terms that are up to $\mathcal{O}\left(\Delta t^{2}\right)$ once we take expectations for the fourth order term in the Taylor expansion is

$$
\frac{3 \Delta t^{2} \sigma^{4}}{24} \partial_{p}^{4} \phi
$$

Thus for the symplectic Euler we have

$$
\begin{aligned}
\mathbb{E}(\phi(p(\Delta t), q(\Delta t)) \mid(p(0), q(0)) & =(p, q))=\Delta t \mathcal{L}_{0} \phi-\Delta t^{2}\left(\gamma p+V^{\prime}(q)\right) \partial_{q} \phi \\
& \left.+\frac{\Delta t^{2}}{2}\left(p^{2} \partial_{q}^{2} \phi+2\left(\sigma^{2}-p\left(\gamma p+V^{\prime}(q)\right)\right) \partial_{p} \partial_{q} \phi+\left(\gamma p+V^{\prime}(q)\right)^{2}\right) \partial_{p}^{2} \phi\right) \\
& +\frac{\Delta t^{2}}{6}\left(3 \sigma^{2} p \partial_{q} \partial_{p}^{2} \phi-3 \sigma^{2}\left(\gamma p+V^{\prime}(q)\right) \partial_{p}^{3} \phi\right) \\
& +\frac{3 \Delta t^{2} \sigma^{4}}{24} \partial_{p}^{4} \phi
\end{aligned}
$$


B.2 1st order Lie-Trotter splitting method. We present the calculations needed to find $\mathcal{A}_{1}$ for the symplectic Euler in greater detail. In doing this is useful to write equation (4.21) in the following way

$$
\begin{aligned}
& q(\Delta t)=q+\Delta t p-\gamma \frac{\Delta t^{2}}{2} p-\Delta t^{2} V^{\prime}(q)+\Delta t \beta \xi+\mathcal{O}\left(\Delta t^{3}\right), \\
& p(\Delta t)=\left(1-\gamma \Delta t+\frac{1}{2} \gamma^{2} \Delta t^{2}\right) p-\Delta t V^{\prime}(q)+\beta \xi+\mathcal{O}\left(\Delta t^{3}\right) .
\end{aligned}
$$

We now need to take the Taylor expansion up to the 4-th order and then take conditional expectations. In doing this it is very useful to express the magnitude of the coefficient $\beta$. More precisely, it is not difficult to see that

$$
\beta^{2}=\sigma^{2}\left(\Delta t-\gamma \Delta t^{2}\right)+\mathcal{O}\left(\Delta t^{3}\right)
$$

Using this it together with the expression (B.1) we obtain

$$
\begin{aligned}
\mathbb{E}(\phi(p(\Delta t), q(\Delta t)) \mid & (p(0), q(0)))=(p, q))=\phi(p, q)+\Delta t \mathcal{L}_{0} \phi \\
- & \Delta t^{2}\left[\left(\gamma p+V^{\prime}(q)\right) \partial_{q} \phi-\frac{\gamma^{2}}{2} p \partial_{p} \phi\right] \\
+ & \frac{\Delta t^{2}}{2}\left(p^{2} \partial_{q}^{2} \phi+2\left(\sigma^{2}-p\left(\gamma p+V^{\prime}(q)\right)\right) \partial_{p} \partial_{q} \phi+\left(\gamma p+V^{\prime}(q)\right)^{2} \partial_{p}^{2} \phi\right) \\
+ & \frac{\Delta t^{2}}{6}\left(3 \sigma^{2} p \partial_{q} \partial_{p}^{2} \phi-3 \sigma^{2}\left(\gamma p+V^{\prime}(q)\right) \partial_{p}^{3} \phi\right)+\frac{3 \Delta t^{2} \sigma^{4}}{24} \partial_{p}^{4} \phi .
\end{aligned}
$$

Appendix C. In this appendix we present some calculations needed for the material presented in Section 7.2. More precisely we present the calculations needed to find $\mathcal{A}_{1}$ for the first order integrated Euler method. In doing this is useful to write equation (6.13) in the following way

$$
\begin{aligned}
& q(\Delta t)=q+\Delta t p-\gamma \frac{\Delta t^{2}}{2} p-\Delta t^{2} V^{\prime}(q)+\alpha \xi+\delta \psi+\mathcal{O}\left(\Delta t^{3}\right) \\
& p(\Delta t)=\left(1-\gamma \Delta t+\frac{1}{2} \gamma^{2} \Delta t^{2}\right) p-\left(\Delta t-\gamma \Delta t^{2}\right) V^{\prime}(q)+\beta \xi+\mathcal{O}\left(\Delta t^{3}\right) .
\end{aligned}
$$

We now need to take the Taylor expansion up to the 4-th order and then take conditional expectations. In doing this it is very useful to express the magnitude of the coefficients $\alpha, \beta, \delta$. More precisely, it is not difficult to see that

$$
\begin{aligned}
\alpha^{2}+\delta^{2} & =\mathcal{O}\left(\Delta t^{3}\right), \\
\alpha \beta & =\frac{\sigma^{2}}{2} \Delta t^{2}+\mathcal{O}\left(\Delta t^{3}\right), \\
\beta^{2} & =\sigma^{2}\left(\Delta t-\gamma \Delta t^{2}\right)+\mathcal{O}\left(\Delta t^{3}\right) .
\end{aligned}
$$

Using this it together with the expression (B.1) we obtain

$$
\begin{aligned}
\mathbb{E}(\phi(p(\Delta t), q(\Delta t)) \mid & (p(0), q(0)))=(p, q))=\phi(p, q)+\Delta t \mathcal{L}_{0} \phi \\
- & \Delta t^{2}\left[\left(\frac{\gamma p}{2}+V^{\prime}(q)\right) \partial_{q} \phi-\frac{\gamma^{2}}{2} p \partial_{p} \phi\right] \\
+ & \frac{\Delta t^{2}}{2}\left(p^{2} \partial_{q}^{2} \phi+\left(\sigma^{2}-2 p\left(\gamma p+V^{\prime}(q)\right)\right) \partial_{p} \partial_{q} \phi+\left(\gamma p+V^{\prime}(q)\right)^{2} \partial_{p}^{2} \phi\right) \\
+ & \frac{\Delta t^{2}}{6}\left(3 \sigma^{2} p \partial_{q} \partial_{p}^{2} \phi-3 \sigma^{2}\left(\gamma p+V^{\prime}(q)\right) \partial_{p}^{3} \phi\right)+\frac{3 \Delta t^{2} \sigma^{4}}{24} \partial_{p}^{4} \phi .
\end{aligned}
$$


Appendix D. In this appendix we present the necessary calculations in order to obtain equation (5.7) from (5.6). We start by noting that from the definition of matrix exponential

$$
\left(e^{-A s}\right)^{\prime}=-A e^{-A s}=-e^{-A s} A,
$$

since $A, e^{-A s}$ commute. Thus, if we denote with $J$ the integral term on the left hand side of (5.6) we have

$$
\begin{aligned}
J & =-\int_{0}^{\Delta t} \tilde{A}^{-1}\left(e^{\tilde{A}(\Delta t-s)}\right)^{\prime} \tilde{\Sigma} \tilde{\Sigma}^{T} e^{\tilde{A}^{T}(\Delta t-s)} d s \\
& =-\left[\tilde{A}^{-1} e^{\tilde{A}(\Delta t-s)} \tilde{\Sigma} \tilde{\Sigma}^{T} e^{\tilde{A}^{T}(\Delta t-s)} d s\right]_{0}^{\Delta t}-\int_{0}^{\Delta t} \tilde{A}^{-1} e^{\tilde{A}(\Delta t-s)} \tilde{\Sigma} \tilde{\Sigma}^{T} e^{\tilde{A}^{T}(\Delta t-s)} \tilde{A}^{T} d s,
\end{aligned}
$$

and thus $J$ satisfies the equation

$$
J+\tilde{A}^{-1} J \tilde{A}^{T}=\tilde{A}^{-1} e^{\tilde{A} \Delta t} \tilde{\Sigma} \tilde{\Sigma}^{T} e^{\tilde{A}^{T} \Delta t}-\tilde{A}^{-1} \tilde{\Sigma} \tilde{\Sigma}^{T} .
$$

We now multiply from the left hand side with $\tilde{A}$ to obtain the discrete Lyapunov equation:

$$
\tilde{A} J+J \tilde{A}^{T}=e^{\tilde{A} \Delta t} \tilde{\Sigma} \tilde{\Sigma}^{T} e^{\tilde{A}^{T} \Delta t}-\tilde{\Sigma} \tilde{\Sigma}^{T} .
$$

\section{REFERENCES}

[1] W.-J. Beyn. Numerical methods for dynamical systems. In Advances in numerical analysis, Vol. I (Lancaster, 1990), Oxford Sci. Publ., pages 175-236. Oxford Univ. Press, New York, 1991.

[2] K. Burrage, I. Lenane, and G. Lythe. Numerical methods for second order stochastic equations. SIAM J.Sci.Comp, 29(1):245-246, 2008.

[3] I. T. Drummond, S. Duane, and R. R. Horgan. The stochastic method for numerical simulations: higher order corrections. Nuclear Phys. B, 220(1, FS 8):119-136, 1983.

[4] C. W. Gardiner. Handbook of stochastic methods. Springer-Verlag, Berlin, second edition, 1985. For physics, chemistry and the natural sciences.

[5] D. T. Gillespie. The chemical langevin equation. The Journal of Chemical Physics, 113(1):297306, 2000.

[6] E. Hairer, C. Lubich, and G. Wanner. Geometric numerical integration, volume 31 of Springer Series in Computational Mathematics. Springer-Verlag, Berlin, 2002.

[7] D. J. Higham. An algorithmic introduction to numerical simulation of stochastic differential equations. SIAM Review, 43:525-546.

[8] D. J. Higham. An introduction to financial option valuation. pages xxii+273, 2004. Mathematics, stochastics and computation.

[9] Y. Hu, T. Li and B. Min Local truncation error analysis of tau-leaping methods: revisited submitted to Comm. Math. Sci., 2009

[10] A. Iserles. A first course in the numerical analysis of differential equations. Cambridge Texts in Applied Mathematics. Cambridge University Press, Cambridge, 1996.

[11] P. Kloeden and E. Platen. Numerical solution of stochastic differential equations, volume 23 of Applications of Mathematics (New York). Springer-Verlag, Berlin, 1992.

[12] T. Li, A. Abdulle, and W. E. Effectiveness of implicit methods for stiff stochastic differential equations. Commun. Comput. Phys., 3(2):295-307, 2008.

[13] G. N. Milstein. Numerical integration of stochastic differential equations, volume 313 of Mathematics and its Applications. Kluwer Academic Publishers Group, Dordrecht, 1995. Translated and revised from the 1988 Russian original.

[14] G. N. Milstein and M. V. Tretyakov. Stochastic numerics for mathematical physics. Scientific Computation. Springer-Verlag, Berlin, 2004.

[15] B. Oksendal. Stochastic Differential Equations. Sringer, 6th edition, 2003.

[16] H. Owhadi and N. Bou-Rabee. Boltzmann-Gibbs preserving Langevin integrators. preprint, 2008. 
[17] G. A. Pavliotis and A. M. Stuart. Multiscale Methods: Averaging and Homogenization. Springer-Verlag, New York, 2008

[18] G. A. Pavliotis, A. M. Stuart, and K. C. Zygalakis. Calculating effective diffusivities in the limit of vanishing molecular diffusion. J. Comp. Phys, 4(228):1030-1055, 2009.

[19] H. Risken. The Fokker-Planck equation, volume 18 of Springer Series in Synergetics. SpringerVerlag, Berlin, 1989.

[20] A. Rößler. Stochastic Taylor expansions for the expectation of functionals of diffusion processes. Stochastic Anal. Appl., 22(6):1553-1576, 2004.

[21] J. M. Sanz-Serna and M. P. Calvo. Numerical Hamiltonian problems, volume 7 of Applied Mathematics and Mathematical Computation. Chapman \& Hall, London, 1994.

[22] T. Shardlow. Modified equations for stochastic differential equations. BIT, 46(1):111-125, 2006.

[23] D. Talay and L. Tubaro. Expansion of the global error for numerical schemes solving stochastic differential equations. Stochastic Anal. Appl., 8(4):483-509 (1991), 1990.

[24] W. Wang and R. D. Skeel. Analysis of a few numerical integration methods for the Langevin equation. Molecular Physics, 101:2149-2156, 2003.

[25] K. C. Zygalakis. Homogenization for inertial particles. Master's thesis, University of Warwick, 2005.

[26] K. C. Zygalakis. Effective Diffusive Behavior For Passive Tracers and Inertial Particles: Homogenization and Numerical Algorithms, PhD thesis. University of Warwick, Coventry, 2008 . 



\section{RECENT REPORTS}

2009

01/09 A Mass and Solute Balance Model for Tear Volume and Osmolar- Gaffney ity in The Normal And The Dry Eye Tiffany

Yokoi

Bron

02/09 Diffusion and permeation in binary solutions Peppin

03/09 On the modelling of biological patterns with mechanochemical Moreo models: insights from analysis and computation Gaffney

Garcia-Aznar

Doblare

04/09 Stability analysis of reaction-diffusion systems with timem- Madzvamuse dependent coefficients on growing domains

Gaffney

Maini

05/09 Onsager reciprocity in premelting solids

Peppin

Spannuth

Wettlaufer

06/09 Inherent noise can facilitate coherence in collective swarm motion

Yates et al.

07/09 Solving the Coupled System Improves Computational Efficiency of the Bidomain Equations

Southern

Plank

Vigmond

Whiteley

08/09 Model reduction using a posteriori analysis

Whiteley

09/09 Equilibrium Order Parameters of Liquid Crystals in the LaudauDe Gennes Theory

10/09 Landau-De Gennes theory of nematic liquid crystals: the OseenFrank limit and beyond

Majumdar

Majumdar

Zarnescu

11/09 A Comparison of Numerical Methods used for Finite Element Modelling of Soft Tissue Deformation

Pathmanathan

Gavaghan

Whiteley

12/09 From Individual to Collective Behaviour of Unicellular Organisms: Recent Results and Open Problems

Xue

Othmer

Erban

13/09 Stochastic modelling of reaction-diffusion processes: algorithms Erban for bimolecular reactions

Chapman

14/09 Chaste: a test-driven approach to software development for phys-

Pitt-Francis et al. iological modelling 
15/09 Block triangular preconditioners for PDE constrained optimiza- Rees tion

Stoll

16/09 From microscopic to macroscopic descriptions of cell migration on growing domains

Baker

Yates

Erban

17/09 The Influence of Gene Expression Time Delays on GiererMeinhardt Pattern Formation Systems

Seirin Lee

Gaffney

Monk

18/09 Analysis of a stochastic chemical system close to a sniper bifurcation of its mean field model

Erban et al.

Copies of these, and any other OCCAM reports can be obtained from:

Oxford Centre for Collaborative Applied Mathematics

Mathematical Institute

24 - 29 St Giles'

Oxford

OX1 3LB

England

www.maths.ox.ac.uk/occam 